\title{
Testing the NURESIM platform on a PWR main steam line break benchmark
}

\author{
S. Kliem ，Y. Kozmenkov , J. Hadek , Y. Perin , F. Fouquet , F. Bernard , A. Sargeni ，D. Cuervo , \\ A. Sabater , S. Sanchez-Cervera , N. Garcia-Herranz , O. Zerkak , H. Ferroukhi , P. Mala
}

\begin{abstract}
Within the NURESAFE project, a main steam line break benchmark has been defined and solved by codes integrated into the European code platform NURESIM. The paper describes the results of the calculations for this benchmark. Six different solutions using different codes and code systems are provided for the comparison. The quantitative differences in the results are dominated by the differences in the secondary system parameters during the depressurization. The source of these differences comes mainly from the application of different models for the two-phase leak flow available in the system codes. The use of two different thermal hydraulic system codes influences the results more than expected when the benchmark was created. The codes integrated into the NURESIM platform showed their applicability to a challenging transient like a main steam line break.
\end{abstract}

\section{Introduction}

The NURESAFE Collaborative Project aimed at making a new and significant step towards the establishing of a European Reference Simulation Platform. This platform with the name NURESIM has been developed over the last years and provides the following main simulation and related possibilities (Chauliac et al., 2011):

- Accurate representation of physical phenomena in reactor and core physics, two-phase flow thermal hydraulics and fuel modeling

- Multi-scale and multi-physics capabilities of reactor safety computations

- Easy coupling of different codes and solvers by using common data structures and generic functions

- Generic pre-processing and post-processing and supervision functions

- Deterministic and statistical sensitivity and uncertainty analyses
The NURESIM-platform is based on the open software simulation platform SALOME (http://salome-platform.org). The actual list of implemented codes with details of implementation is provided in (Chanaron et al., 2015). Examples of application of the NURESIM platform to different kind of reactor safety problems can be found e.g., in (Jimenez et al., 2015; Bestion, 2017; Grahn et al., 2017).

In continuation of the application of the NURESIM, platform situation targets for different reactor types have been defined. The situation target for the pressurized and the VVER reactors is based on main steam line break (MSLB) benchmarks while an ATWS transient has been selected for the boiling water reactor. The current paper deals with the results of the main steam line benchmark for a pressurized water reactor.

The coupling of $3 \mathrm{D}$ neutron kinetic core models to advanced thermal hydraulic system codes started more than twenty years ago. The very first options of coupling used data exchange between codes by means of files (e.g. Feltus, 1994). Later on, the direct integration of the 3D neutron kinetic core models into the system codes and the corresponding creation of one module for the execution of calculations became a typical type of coupling (e.g. Grundmann et al., 1995). In order to reduce the coupling efforts, system codes were provided with well-defined interfaces for the incorporation of 3D neutron kinetic core models. The system code 
ATHLET (Austregesilo, 2012) is one example, which has been coupled to the neutron kinetic core models KIKO3D (Kereszturi et al., 2003), BIPR8 (Lizorkin et al., 2015) and DYN3D (Rohde et al., 2016) using an identical interface. All these types of coupling (including the NURESIM approach) use the so-called operator splitting approach. Recently, Jacobian-Free Newton Krylov methods started to be developed in order to avoid restrictions imposed by the operator splitting (e.g. Gaston et al., 2009). Such developments are mainly driven by the analysis of Generation-IV reactors. A review of both approaches can be found in (Mylonakis et al., 2014).

The need of more detailed analysis of postulated main steam line break accidents was one of the major drivers for the development of coupling of 3D neutron kinetic core models and thermal hydraulic system codes. The hot zero power MSLB analyses using the STAR and the RETRAN codes reported in (Feltus, 1994) were one of the earliest works in that area. The first international benchmarks for coupled code systems were based on postulated MSLB accidents, the TMI-1 benchmark (Ivanov et al., 2003) for a reactor core with rectangular fuel assembly cross section and the VVER440 benchmark (Kliem et al., 2007) for hexagonal fuel assemblies.

The current MSLB benchmark was defined and calculated within the NURESAFE project with the intention to test the multi-physics features of the NURESIM platform with respect to 3D neutron kinetics of the whole core and system thermal hydraulics and to serve as a basis for the verification and use of the advanced NURESIM features such as embedded hybrid coupling with different resolution in the core, the direct coupling with computational fluid dynamics codes in the pressure vessel and in the core (Grahn et al., 2017) or the envisaged coupling with fuel performance codes.

In chapter 2, the description of the benchmark is shortly outlined. Chapter 3 gives an overview on the used codes for the solu- tion of the different parts of the benchmark. In chapter 4 the models developed for the solution of the benchmark are described and chapter 5 contains the comparison between the different platform codes.

\section{Benchmark specification}

A benchmark for the evaluation of the possibilities of codes integrated into the NURESIM platform was defined. It concerns a main steam line break (MSLB) in a pressurized water reactor. The benchmark specification is given below.

The reference reactor is the 4-loop Westinghouse PWR ZION. The reactor core configuration includes fuel assemblies (FAs) of four different types (MOX and UOX FAs, each with two different initial enrichments). Their geometries and initial compositions given in (Kozlowski and Downar, 2003) have been used in preparation of the cross-section library for benchmark simulations (see Section 4.1 of Chapter 4 ). To maximize the reactivity effect of the core overcooling due to the MSLB, the transient is assumed to start at the end of cycle (EOC) when the boron content of coolant is negligible. One cycle calculation has been performed under nominal conditions with CRONOS2 (see Section 3.2.1) to compute the EOC state (radial and axial burnup distributions) (Bernard et al., 2014). The resulting scheme of reactor core model configuration and control rod locations is given in Fig. 2.1 (radial map and axial burnup profile).

The initial steady-state corresponds to the hot zero power (HZP) conditions at the end of a fuel cycle (EOC) summarized in Table 2.1.

A double-ended MSLB at the outlet nozzle of the steam generator (SG) in the first reactor loop is assumed to be the initial event of the accident. The break size is the actual size of the main steam line (MSL) with the diameter of $0.89 \mathrm{~m}$. The flow limiter with the cross

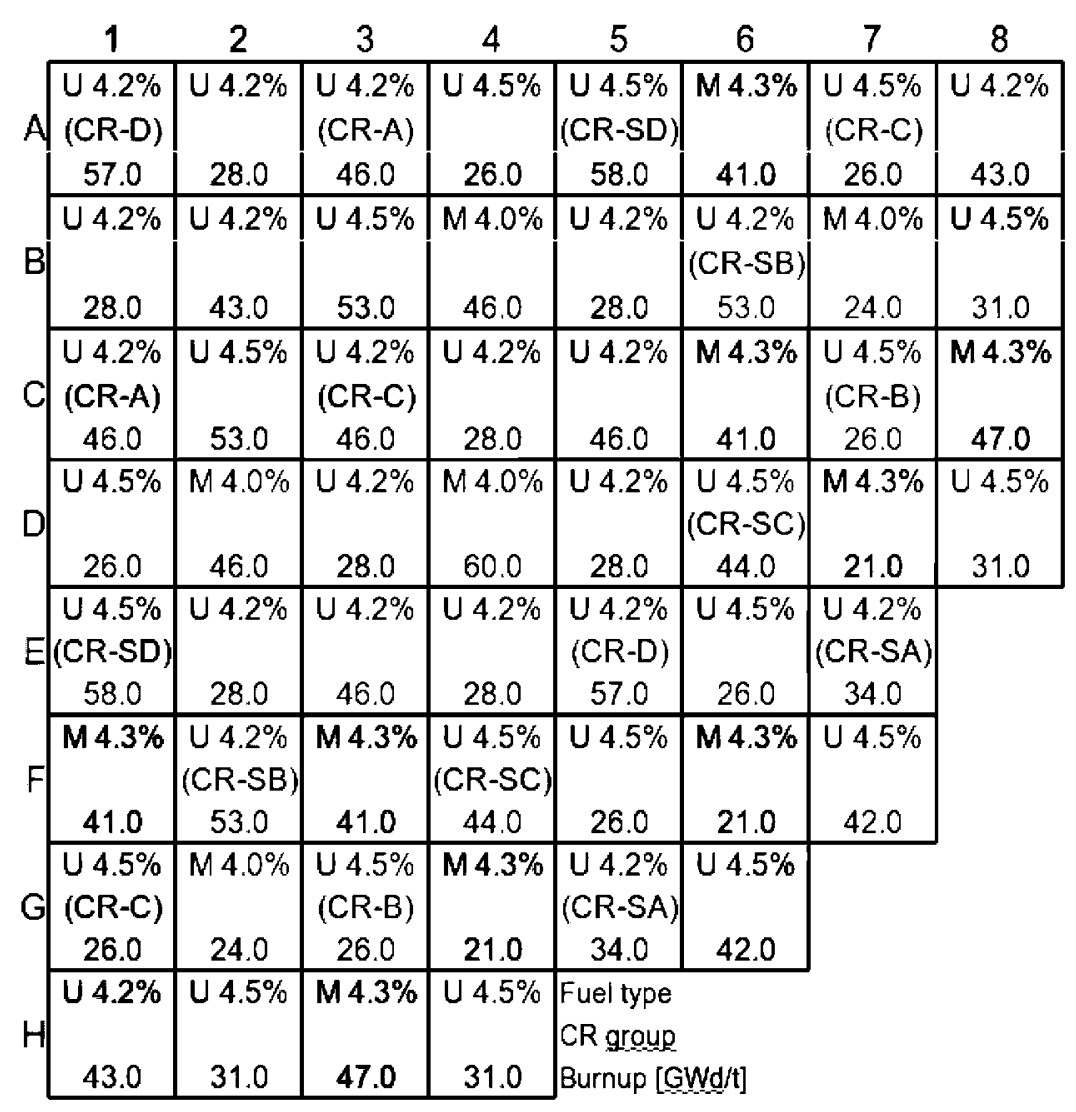

\begin{tabular}{|c|c|}
\hline$Z(m)$ & $\begin{array}{l}\text { Axial Bumup } \\
\text { Ratio }\end{array}$ \\
\hline \multirow{2}{*}{$\begin{array}{l}1.8288 \\
1.6020\end{array}$} & 0.84 \\
\hline & 0.93 \\
\hline 1.2573 & 1.00 \\
\hline \multirow[t]{2}{*}{0.4572} & \\
\hline & 1.03 \\
\hline \multirow[t]{2}{*}{-0.2286} & \\
\hline & 1.05 \\
\hline \multirow{2}{*}{$\begin{array}{l}-1.3716 \\
-1.4859\end{array}$} & 103 \\
\hline & 1.00 \\
\hline-1.6020 & 0.93 \\
\hline-1.8288 & \\
\hline
\end{tabular}

Fig. 2.1. Reactor $1 / 4$ core map (fuel assembly type, initial enrichment, mean burnup at EOC, and location of control rods) and axial burnup profile. 
Table 2.1

Initial steady-state conditions.

\begin{tabular}{ll}
\hline Parameter, units & Value \\
\hline Reactor power at EOC, W & 1000 \\
Decay heat power, W & 0 \\
Boron concentration in the primary coolant, ppm & 0 \\
Shutdown margin ${ }^{1}, \%$ & 1 \\
Pressurizer steam pressure, MPa & 15.44 \\
Pressurizer water level (local length coordinate), $\mathrm{m}$ & 8.8 \\
Primary system mass/volumetric flow rate (total), $\mathrm{kg} / \mathrm{s} / \mathrm{m}^{3} / \mathrm{h}$ & $17360 /$ \\
& 82427 \\
Primary coolant temperature, ${ }^{\circ} \mathrm{C}$ & 283.8 \\
Secondary steam pressure, MPa & 6.72 \\
Secondary coolant level in steam generators (local length & 11.49 \\
$\quad$ coordinate), $\mathrm{m}$ & \\
Feed water (FW) mass flow to steam generators, $\mathrm{kg} / \mathrm{s}$ & 0 \\
\hline
\end{tabular}

1 In the benchmark definition it is recommended to modify the production operator at the beginning of the transient in such a way that the required subcritical level is reached and keep the modification constant throughout the whole transient. Deviations from this approach are discussed in the corresponding description of models.

flow area of $1400 \mathrm{~cm}^{2}$ is located upstream of the MSL break. This flow limiter as well as the flow limiters in intact loops must be modeled. Until main steam isolation valve (MSIV) closure upon appropriate signal (see the accident scenario assumptions below) the reverse steam flow coming from the intact SGs to the break can be calculated on a realistic way, according to the geometry of pipes (including main steam header) and corresponding flow resistances. Or it can be also calculated in a more conservative way, ignoring these flow resistances, and only accounting for the flow limiters at the exits of intact SGs; this results in easier depressurization of the intact SGs and in faster cooldown of the reactor coolant system (RCS) and the core.

The MSLB scenario assumptions are:

- Stuck control rod (CR) with a highest worth belongs to the core coldest area

- Fuel assembly with the stuck CR has a high enthalpy peaking factor (FDH) value

- All main coolant pumps remain in operation during the whole transient

- Pressurizer heaters are switched off during the whole transient

- No coolant injection from hydraulic accumulators

- Closure of MSIVs starts when the main steam header (MSH) pressure drops below 5.5 MPa

- The duration of MSIVs full closure is $10 \mathrm{~s}$ (linear reduction of the flow area)

- FW pumps are switched on at $0.0 \mathrm{~s}$ of the accident

- FW mass flow rates are: $83.3 \mathrm{~kg} / \mathrm{s}$ (to SG 1) and $5.6 \mathrm{~kg} / \mathrm{s}$ (to SGs 2-4)

- FW temperature is $10^{\circ} \mathrm{C}$

- FW pumps are switched off by the signal of safety injection system (SIS) actuation

- The signal for SIS actuation is generated when the pressure in the reactor upper plenum drops below $12.05 \mathrm{MPa}$

- SIS is not actuated (boron is not injected to the primary system)
For the coolant mixing in the lower plenum (LP) a model based on experimental data obtained at the coolant mixing test facility ROCOM (Kliem et al., 2008) was considered. This model provides mixing coefficients distributing the cold legs flows onto the fuel assembly inlets.

For stand-alone core models the transient boundary conditions (assembly-wise time dependences of inlet coolant enthalpy/temperature and mass flow rate as well as the pressure at the core outlet) are calculated by a coupled code.

The sequence of events corresponding to the above described accident scenario is as follows:

- Double ended MSL break (initiating event)

- Start of FW supply to SGs

- Core criticality reached

- Maximum overcriticality reached

- Signal for SIS actuation/End of FW supply to SGs

- Maximum core power reached

- Minimum coolant collapsed level in pressurizer reached

- Return to subcritical state

- Core power drops below $1 \mathrm{MW} /$ End of the transient simulation

Selected results of steady-state/HZP and transient calculations (core power and reactivity, primary and secondary thermal-hydraulic parameters, etc.) are included into the comparison, which is presented in Chapter 5 .

\section{Computation tools}

\subsection{Overview on used codes and settings}

Table 3.1 gives an overview on the codes used the different participants for the calculation of the benchmark.

In the following, the corresponding codes are described shortly.

\subsection{Thermal hydraulic system codes}

\subsubsection{ATHLET}

The thermal-hydraulic computer code ATHLET (Analysis of Thermal hydraulics of LEaks and Transients) is developed by the Gesellschaft fuer Anlagen- und Reaktorsicherheit (GRS) mbH for the analysis of anticipated and abnormal plant transients, small and intermediate leaks as well as large breaks in light water reactors (Austregesilo, 2012). The aim of the code development is to cover the whole spectrum of design basis and beyond design basis accidents (without core degradation) for PWRs, VVERs and BWRs with only one code. The main code features are: advanced thermal hydraulics, modular code architecture, separation between physical models and numerical methods, pre- and post-processing tools and portability. The code offers the possibility of choosing between models for the simulation of fluid dynamics. The first option is a five-equation model with separate conservation equations for liquid and vapour mass and energy, and a mixture equation, accounting for the thermal and mechanical non-equilibrium, and including a mixture level tracking capability. Further, a complete two-fluid

Table 3.1

Overview on codes used by the different participants.

\begin{tabular}{|c|c|c|c|c|c|c|}
\hline & HZDR & GRS & IRSN & UJV & UPM & PSI \\
\hline System Thermal hydraulics & ATHLET & ATHLET & CATHARE & ATHLET & Time dependent boundary conditions & Time dependent boundary conditions \\
\hline Core Thermal hydraulics & FLOCAL & ATHLET & FLICA & FLOCAL & COBRA-TF & SIMULATE-3K ${ }^{1}$ (S3K) \\
\hline Neutron kinetics & DYN3D & DYN3D & CRONOS2 & DYN3D & COBAYA3 & SIMULATE-3K (S3K) \\
\hline
\end{tabular}

\footnotetext{
1 The code SIMULATE-3K is not integrated into the NURESIM platform. The results provided by this code are included as an additional variant.
} 
model with separate conservation equations for liquid and vapour mass, energy, and momentum is available. Moreover, both fluid dynamic options allow for the simulation of non-condensable gases on the basis of the ideal gas formulation. Additional mass conservation equations can be included for the description of boron transport within a coolant system as well of the transport and release of nitrogen dissolved in the liquid phase of the coolant. The time integration of the thermal fluid dynamics is performed with a general solver for ordinary differential equations called FEBE (Forward-Euler, Backward-Euler).

For the simulation of heat conduction a one-dimensional heat conductor module (HECU) is available. This model can simulate the one-dimensional temperature profile and heat conduction in plates, hollow and full cylinders in radial direction. The created heat conduction objects can be coupled on left and/or right side of thermal fluid objects by consideration of the energy transport between the heat conductor surface and the surrounding fluid. A heat transfer package which covers a wide range of single phase and two-phase flow conditions is available inside the module. Correlations for critical heat flux and minimum film boiling temperature are included; evaporation and condensation directly at heating surfaces are calculated. A quench front model for bottom and top reflooding is also available.

The code development is accompanied by a systematic and comprehensive validation program. A large number of integral experiments and separate effect tests, including the major International Standard Problems, have been calculated by GRS and by independent organizations. ATHLET is being applied by numerous institutions in Germany and abroad.

\subsubsection{CATHARE2}

CATHARE2 (Code for Analysis of Thermal-hydraulics during an Accident of Reactor and safety Evaluation) is a two-phase thermal-hydraulic simulator in development since 1979 as part of an agreement between the CEA, EDF, AREVA and the IRSN. The code is used, in particular, in pressurized water reactor safety analyses, the verification post-accidental operating procedures and in research and development (Geffraye et al., 2011).

The CATHARE2 simulator has a modular structure capable of operating in $\mathrm{OD}$ (volumes), $1 \mathrm{D}$ (pipes) or 3D (vessel and boundary conditions), connected to each other by junctions. Other submodules feature pumps and turbo-machines, control valves, Tjunctions, sinks and sources, break and many others.

CATHARE2 is capable of modeling any type of PWR reactor, several types of integral (including BETHSY and LOFT) and many single-effect test facilities (CANON, ECTHOR, MARVIKEN, PERICLES, etc.). It also contains a module being capable of simulating the behavior of a nuclear reactor containment vessel in the event of a break in either the primary or secondary circuit.

The code is based on a two-phase model with six equations (conservation of mass, energy and quantity of movement for each phase). The discretization of all the terms of the equations is fully implicit in 1D and OD modules and semi-implicit in 3D elements including inter-phase exchange, pressure and convection terms, and the resulting non-linear equations are solved using an iterative Newton solver. The code allows efficient use of several processors in parallel. This robust and efficient method provides a good compromise between accuracy and computing cost.

The successive versions of the code were validated in a two-step process: validation in separate-effect experiments (CANON, ECTHOR, MARVIKEN, PERICLES, etc.) followed by validation of the overall behavior of the code in integral experiments (particularly BETHSY and LOFT).

CATHARE2 is capable of simulating the physical phenomena that occur during a loss of coolant accident (small and large break LOCA), steam generator tube rupture (SGTR), feed water line break
(FWLB), residual heat removal failure (RHR), or steam line break (SLB). CATHARE2 has also been integrated into the IRSN' HEMERA chain and into the IRSN' SOFIA accidental simulator for the French reactors. This simulator is used to train specialists, prepare emergency management exercises, and to carry out studies in support of reactor assessments.

\subsection{Thermal hydraulic and neutron kinetic core simulators}

\subsubsection{CRONOS2}

The CRONOS2 code is a component of the CEA/SAPHYR codes system, devoted to core computations (Lautard et al., 1999). CRONOS2 allows solving the diffusion/simplified transport equations in a multi-group energy modeling with a 3D geometry (Cartesian and hexagonal). It allows, as well, the resolution of the 3D and $O D$ kinetic equations and to follow the depletion of actinides. The Xe and $\mathrm{Sm}$ chains are represented explicitly.

CRONOS2 makes available some different solvers to solve the diffusion/transport equations. IRSN uses currently a mixed dual finite elements method called MINOS. The MINOS method (Lautard et al., 1999 and Lewis, 1999) solves the dual form of the P1 transport equation (in each energy group):

$\vec{\nabla} \cdot \vec{p}+\sigma \varphi=S$

$\vec{\nabla} \varphi+D^{-1} \vec{p}=\vec{Q}$

by transforming it in a variational problem with unknown $(\varphi, \vec{p})$, flux and current. The discretization of the finite elements depends on the choice of an approximate subspace (finite-dimensional subspace composed of piecewise polynomial functions) then, using the Ritz-Galerkin method (projection into the approximate space itself), a set a discrete equations is obtained. For the Cartesian geometry, the Raviart-Thomas-Nedelec elements are used.

Moreover, CRONOS2 owns simplified heat conduction and thermal hydraulic models to calculate the fuel temperature inside a 'mean' fuel pin (a pin with the mean value of the pin power) and the water density distribution by mesh inside a channel (a channel by assembly). The fuel temperature is used to calculate the Doppler temperature (Rowlands' formula): $4 / 9 T_{\text {center }}+5 / 9 T_{\text {periph }}$.

CRONOS2 is provided with cross sections prepared by means of the lattice code APOLLO2 (Sanchez, 1988).

\subsubsection{DYN3D}

The reactor dynamics code DYN3D is a three-dimensional bestestimate tool for simulating steady states and transients of Light Water Reactors (LWRs), which has been developed at the Helmholtz-Zentrum Dresden-Rossendorf (HZDR). It consists of a neutron kinetic and a thermal hydraulic model.

The neutron kinetic model is based on the solution of the three dimensional multi-group neutron diffusion equation by nodal expansion methods. It is assumed that the macroscopic cross sections are spatially constant in a node being the part of a fuel assembly. Different approaches for the nodal flux expansion are used for Cartesian and hexagonal fuel assembly geometry (Rohde et al., 2016). In the case of Cartesian geometry, the three-dimensional diffusion equation of each node is transformed into onedimensional equations in each direction $\mathrm{x}, \mathrm{y}, \mathrm{z}$ by transverse integrations. The equations are coupled by the transverse leakage terms. In each energy group, the one-dimensional equations are solved with the help of flux expansions in polynomials up to 2 nd order and exponential functions being the solutions of the homogeneous equation. The fission and the scattering sources as well as the leakage terms are approximated by polynomials. In the case of hexagonal fuel assemblies, two different geometrical options are 
available. The diffusion equation in the node is transformed into a two-dimensional equation in the radial plane and a onedimensional equation in the axial direction. The two equations are again coupled by the transverse leakage terms.

In the steady state, the homogeneous eigenvalue problem or the heterogeneous problem with given source is solved. An inner and outer iteration strategy is applied. The outer iteration (fission source iteration) is accelerated by Chebychev extrapolation. The steady-state iteration technique is applied for the calculation of the initial critical state, the depletion calculations and the Xe and Sm dynamics. Concerning reactivity transients, an implicit difference scheme with exponential transformation is used for the time integration over the neutronic time step. The heterogeneous equations obtained after performing the exponential transformation for each time step are solved by an inner and outer iteration technique similar to the steady state.

The thermal hydraulic model of the reactor core and the fuel rod model are implemented in the module FLOCAL (Manera et al., 2005), which is a part of DYN3D. The reactor core is modeled by parallel coolant channels, which are coupled hydraulically by the condition of equal pressure drop over all core channels. Thermal hydraulic boundary conditions for the core like coolant inlet temperature, pressure, and coolant mass flow rate or pressure drop must be given as input for DYN3D. FLOCAL comprises a one- or two-phase coolant flow model on the basis of four differential balance equations for mass, energy and momentum of the two-phase mixture and the mass balance for the vapor phase allowing the description of thermodynamic non-equilibrium between the phases as well as a heat transfer regime map from one-phase liquid up to post-critical heat transfer regimes and superheated steam.

The 1D fuel rod model includes heat conduction in the fuel and in the cladding and heat transfer through the gas gap between them (Rohde, 2001). Besides the fuel temperature, which is a reactivity feedback parameter for the neutron kinetics, and its radial distribution the model provides data for safety estimation like fuel enthalpy, maximum cladding temperature, cladding oxide layer thickness and cladding mechanical failure.

\subsubsection{COBAYA3}

COBAYA3 is a multi-scale neutron diffusion code for LWR core analysis developed at UPM. It is able to compute steady state and space-time kinetics problems. It solves the 3D multi-group neutron diffusion equation for both Cartesian rectangular and hexagonal geometries.

COBAYA3 includes a nodal and a pin-by-solver, allowing analysis at both coarse and fine mesh scales.

The nodal solver implemented is called ANDES -Analytical Nodal Diffusion Equation Solver- (Lozano et al., 2008). It can be used stand-alone to perform nodal full core calculations, or as an accelerating module for the pin-by-pin solver. ANDES is based on the Analytic Coarse Mesh Finite Difference Method (ACMFD) (Aragones et al., 2007) which basically consists of two steps. The first step is a diagonalization of the multi-group diffusion matrix to transform the coupled multi-group diffusion equation into a set of uncoupled equations; the second step is a transverse integration procedure to transform the $\mathbf{n}$-dimensional equation into $\mathbf{n}$ one-dimensional equations in order to find an analytic 1D solution on each dimension. ANDES has been extensively validated for a number of numerical benchmarks (Lozano et al., 2010).

The pin-by-pin solver included in COBAYA3 is based on the transport-corrected fine-mesh finite-differences (FMFD) diffusion method. This solver can be used to solve full core problems in two different ways: defining one calculation domain or through domain decomposition by alternate core dissections. While the first method is suitable in shared memory systems, the second one is more convenient for distributed memory systems where every domain can be solved in a different machine. A nodal acceleration via ANDES can be performed in order to speed up the full core solution process.

When coupled to a thermal-hydraulic code, COBAYA3 can be used to analyse steady-state problems, with eigenvalue or critical boron search, and transient problems originated by changes in control bank positions, boron concentration or coolant conditions. Transients can be performed starting from a critical or a subcritical steady-state. In the latter case, COBAYA includes a method to determine a virtual external neutron source (Jimenez et al., 2015), intended to find a steady state solution of the subcritical system. Also, the capability of initiating the transient from a fixed target reactivity value has been implemented recently to exactly fulfill an imposed shutdown margin.

\subsubsection{FLICA-IV}

FLICA-IV is a three-dimensional thermal-hydraulic code dedicated to two-phase calculations required for the design and the safety analysis of nuclear reactor cores (Toumi et al., 2000). It has been developed by the CEA and it is integrated in the CEA/SAPHYR codes system.

The two-phase flow model in FLICA-IV consists of a mixture mass conservation equation, a mixture momentum balance equation, a mixture energy balance equation and a mass phase equation to calculate thermal non-equilibrium or sub-cooled boiling flows. As a constitutive equation, a drift-flux model is included to model the relative velocity between the phases. FLICA-IV is able to treat both a homogeneous equilibrium model that a two-fluid models involving unequal pressure for each phase.

The numerical method in FLICA-IV is based on an approximate Riemann solver and it requires the solution of a one-dimensional Riemann problem at cell interfaces to define, on a non-staggered grid, backward and forward differences of the spatial derivatives.

FLICA-IV may also compute the temperature field in the nuclear fuel (rods and flat plates) for transient and steady state analysis. In FLICA-IV an additional equation relative to solute transport in also available to simulate, for example, boron transport in PWR cores during transients and accidents.

FLICA-IV has had an extended validation program, in order to optimize the closure models in accordance with the comparisons between computed and experimental data. The experimental program has been divided into single-phase flow validation (using the CEA facilities AGATE and HERMES) and two-phase flow validation (using the CEA facility GRAZIELLA).

The 'standard' FLICA-IV modeling, in IRSN consists of the simulation of one channel by assembly. In addition, FLICA-IV has the capability to discretize an assembly at a pin-by-pin scale ('hybrid' modeling). With this option, two FLICA-IV codes run at the same time: the 'standard' FLICA runs and provides boundary conditions to the 'hybrid' FLICA-IV part. This configuration is used in the coupled calculations with CRONOS2.

\subsubsection{COBRA-TF}

COBRA-TF is a two-fluid, three-field thermal-hydraulic code for simulation of LWR cores that solves the conservation equations for mass, momentum and energy for the two phases (Salko and Avramova, 2015). It also includes mass and momentum equations for the entrainment droplets in the vapor phase. The equations can be solved in three-dimensions and it has great flexibility to define radial and axial nodalization. The code includes also the transfer of energy in the nuclear rod providing the temperature profile in the rod and clad at each rod and axial level.

The code has been developed and improved by the Reactor Dynamics and Fuel Modeling Group (RDFMG) at the North Carolina State University (NCSU) as part of the U.S. DOE CASL project. It can be run in serial and parallel modes. Another improvement has been 
the development of models, enhancing computational efficiency as well as improving software quality and associated Quality Assurance.

The integration in the NURESIM platform was performed by GRS. It allows using the coolant centered model for subchannel representation at pin level, but also the average channel model to represent the whole assembly by only one radial node.

\subsubsection{SIMULATE-3K}

SIMULATE-3K (S3K) is a best-estimate LWR core dynamics code that on a stand-alone basis is employed for full core coupled neutronics/thermal hydraulics transient analyses. For the neutronics, S3K is based on a 3D two-group neutron diffusion solver, including a 6-group model for delayed neutron precursors. A transverseintegrated nodal method using either polynomial flux expansions or analytical functions for the thermal flux is applied for the spatial integration and the frequency transform method is used for the time integration during the transient. For the thermal hydraulics, a fiveequation drift flux model is employed to solve for 1D nonequilibrium two-phase flow (Grandi, 2011). Through this coupled solver, S3K is designed for general LWR core transient analyses using full core models i.e. with each fuel bundle represented as a single neutronic assembly coupled with an independent single thermal hydraulic flow channel. For boiling water reactors, the S3K core model can be complemented by a thermal hydraulic vessel model.

Based on extensive validation of the latter approach, S3K has been adopted at PSI as the reference code for transient analyses of the Swiss reactors. However, as will be elaborated in subsequent section 4.3.5, an in-house version of S3K has been employed in this study and special adaptations to the standard PSI methodology had to be made in order to closely conform to the benchmark specifications, namely version 2.04.00_MOX4_PSIO2.

\section{Computation models}

\subsection{Cross section library}

A homogenized cross-sections library appropriate for the simulation of the MSLB transient has been generated. This crosssections library is used by all participants in the benchmark.

\subsubsection{Calculation scheme for fuel assemblies}

APOLLO2 (Sanchez, 1988) is the lattice physics code used to compute the two-group homogenized cross-sections and other parameters. A 281-group neutron data library based on JEFF3.1.1 is employed. Fuel assembly transport calculations with reflective boundary conditions are performed applying the options recommended in NURISP Project (Kolev et al., 2011) to produce accurate parameters. The scheme involves:

- Nominal depletion calculations at reference values of state variables (moderator temperature/ density, fuel temperature and boron concentration) in order to obtain the isotopic compositions corresponding to the assembly average burnup values specified in the 3 -D core configuration map. APOLLO2 MOC solver is used for this task.

- Branch calculations at specified burnup values to model instantaneous effects of state variables. It is assumed that control rod insertion turns an assembly type into a different type. An APOLLO2 two-level industrial scheme is used for this task, involving two consecutive steps: i) self-shielding calculation in 281-group energy mesh and multi-cell geometry (using Pij method); and ii) MOC calculation after collapsing to 70-group energy mesh and using a more accurate geometry, well segmented.
The complete set of homogenized data include macroscopic cross sections, discontinuity factors (two types: based on generalized equivalence theory and based on a black-box homogenization) and kinetics parameters (provided in eight delayed groups). Parameters are collapsed in 2 energy groups using the standard $0.625 \mathrm{eV}$ energy cut-off.

Data are provided in NEMTAB-like format, that is, the data dependence on the state variables is specified through a multidimensional table look-up. Core simulators can then use simple linear interpolation schemes to compute appropriate parameters at the required reactor conditions. To minimize the interpolation error, an optimisation process is used to set the grid of state variables values for branch calculations (Sánchez-Cervera et al., 2014a, $2014 \mathrm{~b}$ ). This process refines the grid in the domain where k-eff is more sensitive to cross-section variation, which is assessed by computing adjoint-flux based sensitivity coefficients with COBAYA code. The final state variables distribution is shown in Table 4.1.

APOLLO2 outputs, in Hierarchical Data Format (HDF), require a post-processing tool (SAPHYB-BROWSER) to extract the information to NEMTAB format and compute interface discontinuity factors from transport neutron fluxes and currents.

\subsubsection{Reflector cross-sections}

The procedure to generate reflector cross sections is based on Lefebvre-Lebigot method (Marguet, 2011). For a one-dimensional model of the reflected configuration a transport calculation is performed and a diffusion analytical formulation is derived. The free parameters of the diffusion model are adjusted to achieve, at the fuel/reflector interface, the conservation of flux slopes and spectrum ratio. Here, this method is applied using DRAGON4 code with a very fine spatial meshing. Reflector cross sections are tabulated in boron concentration and no dependence on thermal hydraulics conditions is considered.

\subsection{Modeling of the system}

\subsubsection{HZDR, UJV and GRS}

HZDR, UJV and GRS used the ATHLET code for the simulation of the benchmark. The corresponding input deck for the ATHLET code of the PWR ZION was initially developed by GRS to perform LBLOCA analyses (BEMUSE Phase IV Report, 2008). The reactor core model was based on point kinetics. After required modifications (change of the reactor initial state, disabling the primary and enabling the secondary system leak, etc.) the reactor model was adjusted to fit the MSLB benchmark scenario (Kozmenkov and Kliem, 2013; Kozmenkov and Kliem, 2014).

In the external coupling between DYN3D and ATHLET, the thermal hydraulics model of the whole plant is split into two parts. The first part describes the thermal hydraulics of the reactor core (DYN3D) and the second solves the thermal hydraulics of the rest of the system (ATHLET). The interfaces between them are located at the bottom and the top of the core (Fig. 4.1).

HZDR and UJV used this type of coupling for the calculation of the benchmark.

The so-called internal coupling approach is illustrated in Fig. 4.2. With this approach the ATHLET model covers the whole

Table 4.1

Optimized grid structure for the state variables (120 branch cases).

\begin{tabular}{ll}
\hline State Variable & Data-points \\
\hline Moderator density $\left(\mathrm{kg} / \mathrm{m}^{3}\right)$ & 300382.58465 .15630 .3960 .6 \\
Doppler temperature $(\mathrm{K})$ & 373779.751186 .52000 \\
Moderator temperature $(\mathrm{K})$ & 373495617 \\
Boron concentration $(\mathrm{ppm})$ & 0600 \\
\hline
\end{tabular}




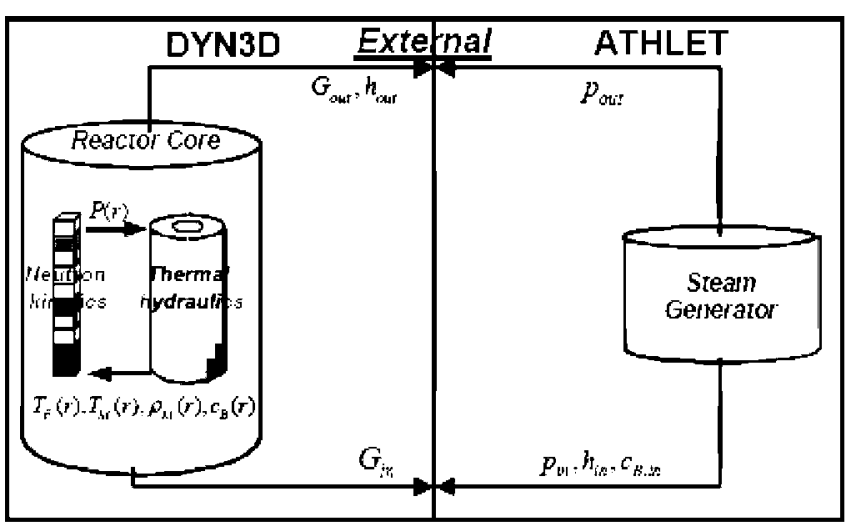

Fig. 4.1. DYN3D/ATHLET external coupling.

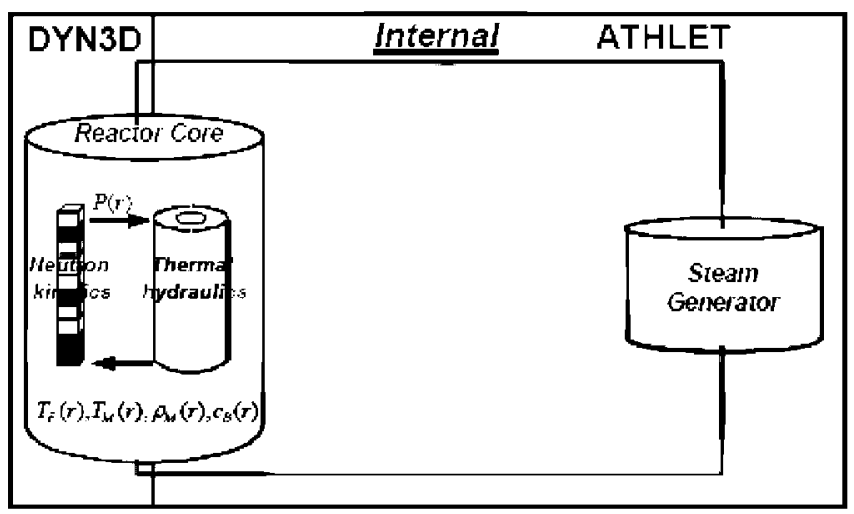

Fig. 4.2. DYN3D/ATHLET internal coupling. thermal hydraulic system including the core. DYN3D uses the thermal hydraulic feedback provided by ATHLET and returns the power. This approach is applied by GRS.

The primary system model (Fig. 4.3) includes the reactor pressure vessel (RPV) with lower and upper plenums, upper head and downcomer as well as four reactor loops with main circulation pumps (MCPs) and SGs of the vertical U-tube design. The pressurizer is connected to the hot leg of the loop with the broken MSL.

The secondary system model (Fig. 4.4) includes four SGs (separators, steam domes, downcomer and riser sections) and four MSLs with main steam isolation valves. The length coordinate of the MSIV location is $13.7 \mathrm{~m}$ from the MSL inlet junction. All main steam lines are connected to the main steam header (not shown in Fig. 4.4). The MSH length and diameter are $10.0 \mathrm{~m}$ and $0.89 \mathrm{~m}$, respectively. The length coordinate of the MSL connection to MSH is $16.2 \mathrm{~m}$ (from the MSL inlet junction). The mass flow rate and temperature of feed water are given as boundary conditions. The data used by the ATHLET model of SG are given in Tables 4.3 and 4.5 .

\subsubsection{IRSN}

IRSN used the CATHARE2 code to model the primary and secondary system of the NPP. Inside the reactor pressure vessel model, the reactor core is modeled by means of the FLICA-IV code. For that purpose, the core is connected by boundary conditions at its inlet and outlet to the CATHARE2 vessel model. The coupling between CATHARE2 and FLICA-IV is working as following:

- CATHARE2 provides the flow rate, enthalpy and boron concentration at the inlet of the core and pressure at the outlet of the core,

- FLICA-IV gives the pressure at the inlet of the core and flow rate, enthalpy and boron concentration at the outlet of the core.

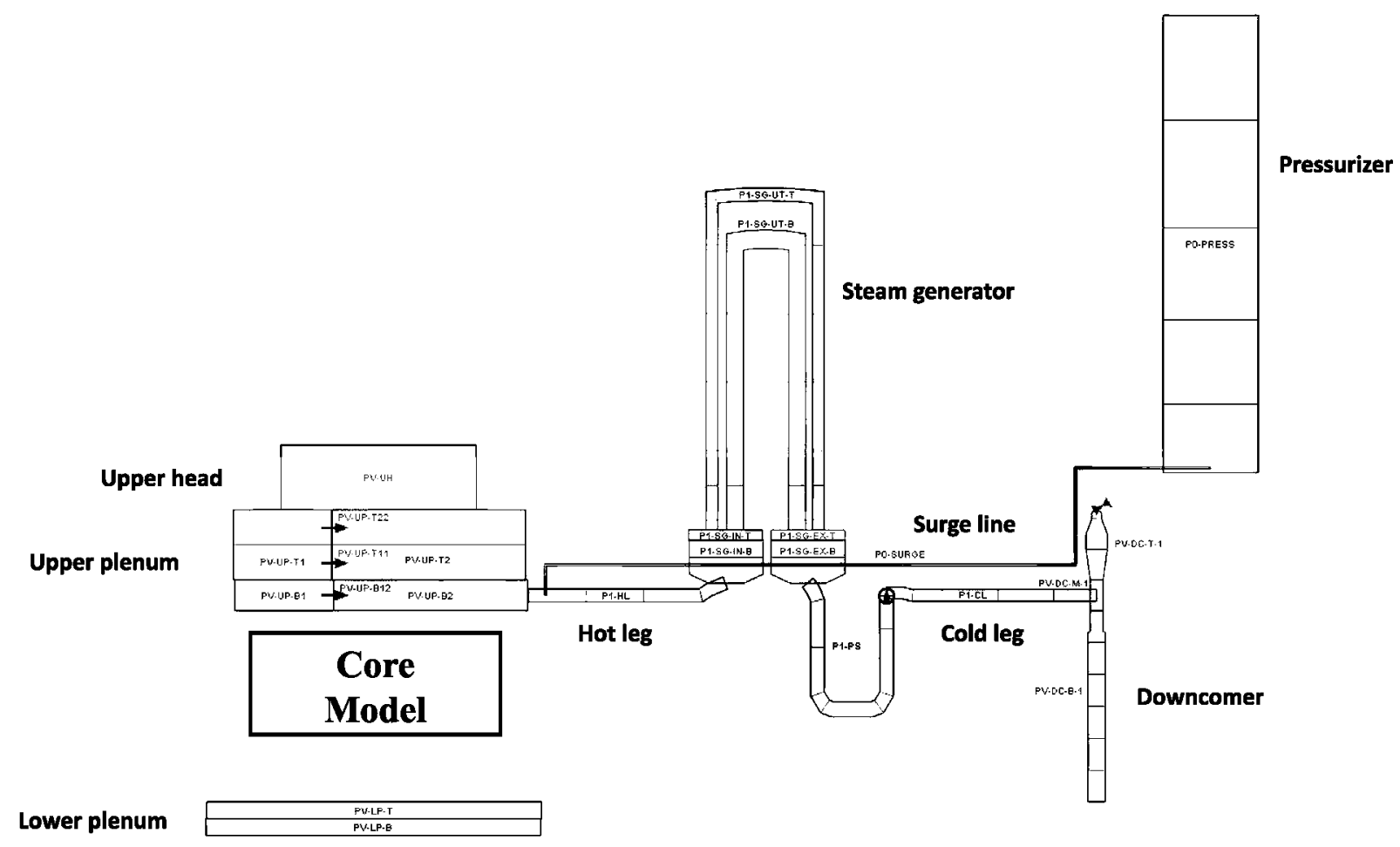

Fig. 4.3. Primary system model. 


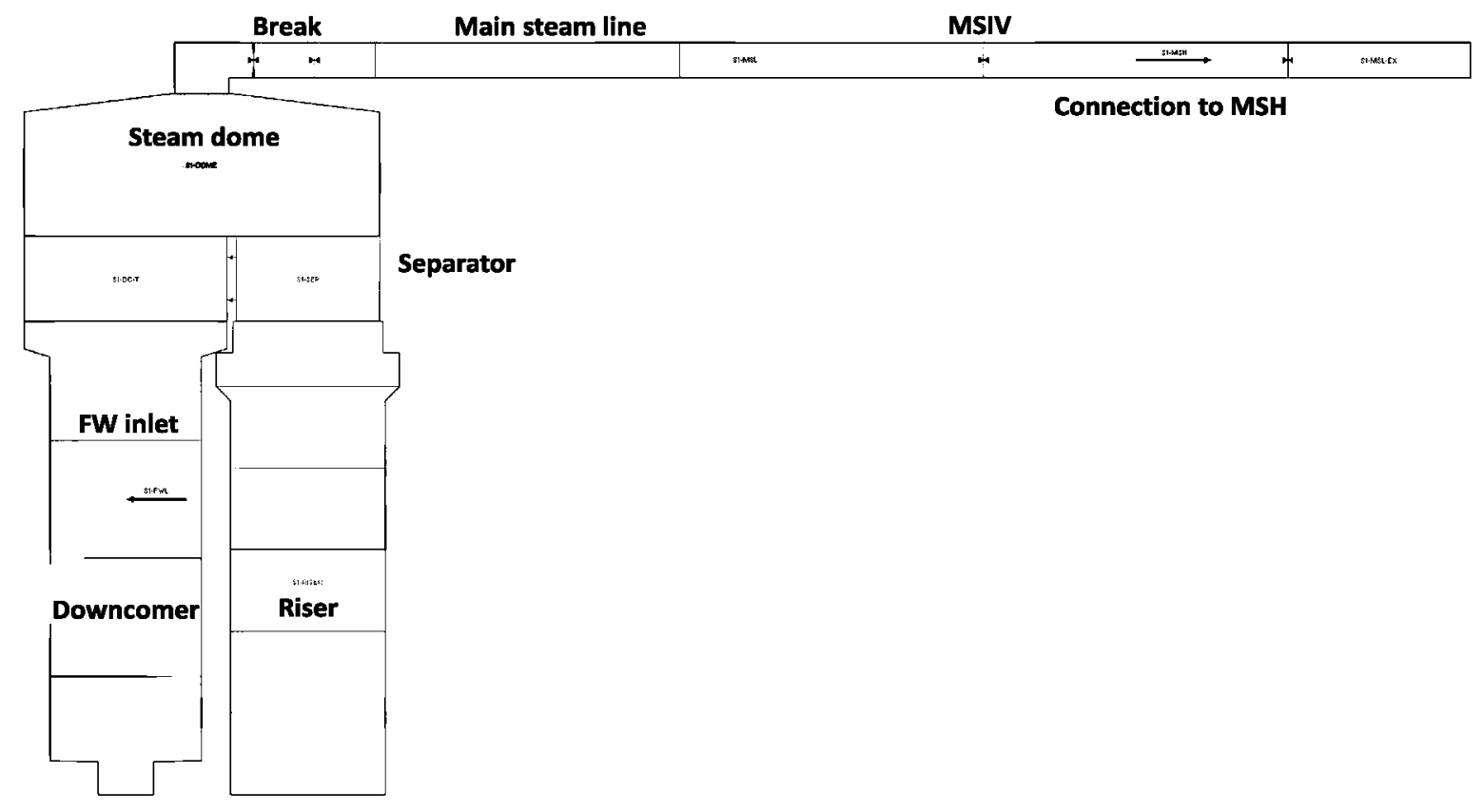

Fig. 4.4. Secondary system model.

In order to be able to taken into account the correct amount of coolant mixing provided by the specification, each element of the vessel was split into four elements. The mixing matrix is connected to the boundary between the CATHARE2 and the FLICA-IV model.

Fig. 4.5 presents the GUITHARE (Graphic User Interface of CATHARE2) sketch of the vessel.

Here after are presented the GUITHARE sketches of the main primary system and secondary circuits corresponding to the CATHARE2 nput deck (Figs. 4.6, 4.7).

The flow limiter is meshing (diminution of the section of the pipe) and the break is represented by a real double ended rupture. It is shown in Fig. 4.8.

\subsection{Modeling of the reactor core}

\subsubsection{IRSN}

The CRONOS2 model, which has been used for the neutronic modeling of the core consists of four meshes ('nodes') in the radial

\section{Table 4.3}

Steam generator geometry data.

\begin{tabular}{lll}
\hline Parameter & ATHLET & Design data \\
\hline Total volume (secondary side), $\mathrm{m}^{3}$ & 165.1 & 166.2 \\
Number of U-tubes & 3388 & 3388 \\
U-tube outer diameter, $\mathrm{m}$ & 0.0222 & 0.0222 \\
U-tube thickness, $\mathrm{m}$ & 0.0013 & 0.0013 \\
Total heat transfer area, $\mathrm{m}^{2}$ & 4612 & 4785 \\
\hline
\end{tabular}

Table 4.4

Steam generator initial conditions.

\begin{tabular}{ll}
\hline Parameter & Value \\
\hline Volume of liquid, $\mathrm{m}^{3}$ & 83.1 \\
Volume of steam, $\mathrm{m}^{3}$ & 82.0 \\
Total mass of secondary coolant, $\mathrm{kg}$ & 64780 \\
Steam dome pressure, $\mathrm{MPa}$ & 6.72 \\
Liquid/steam temperature, ${ }^{\circ} \mathrm{C}$ & 283.1 \\
\hline
\end{tabular}

plane of the assembly and an axial mesh length around $11-12 \mathrm{~cm}$. Each quarter of assembly (the 'node') has its own cross section set that is updated at every change of the thermal-hydraulic node conditions. Both for static and for transient calculations, the CRONOS2 was coupled to the FLICA-IV model in such a way that the four

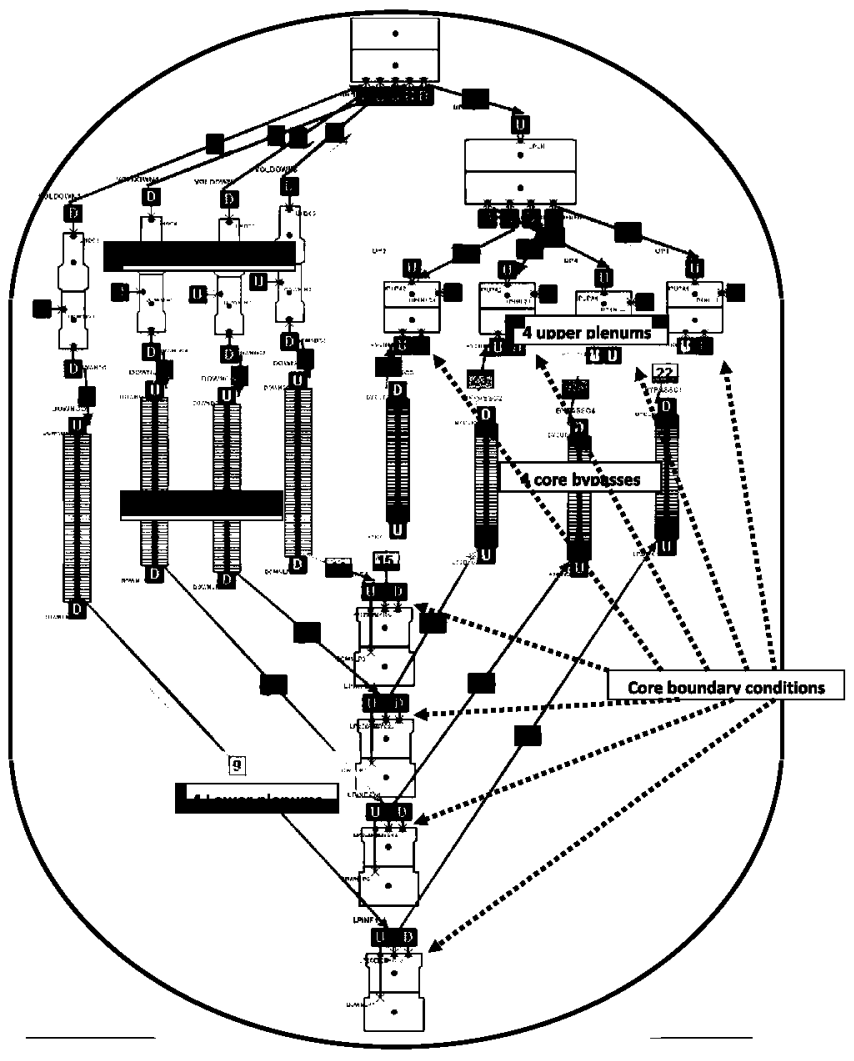

Fig. 4.5. Nodalization of the vessel within CATHARE2 code. 


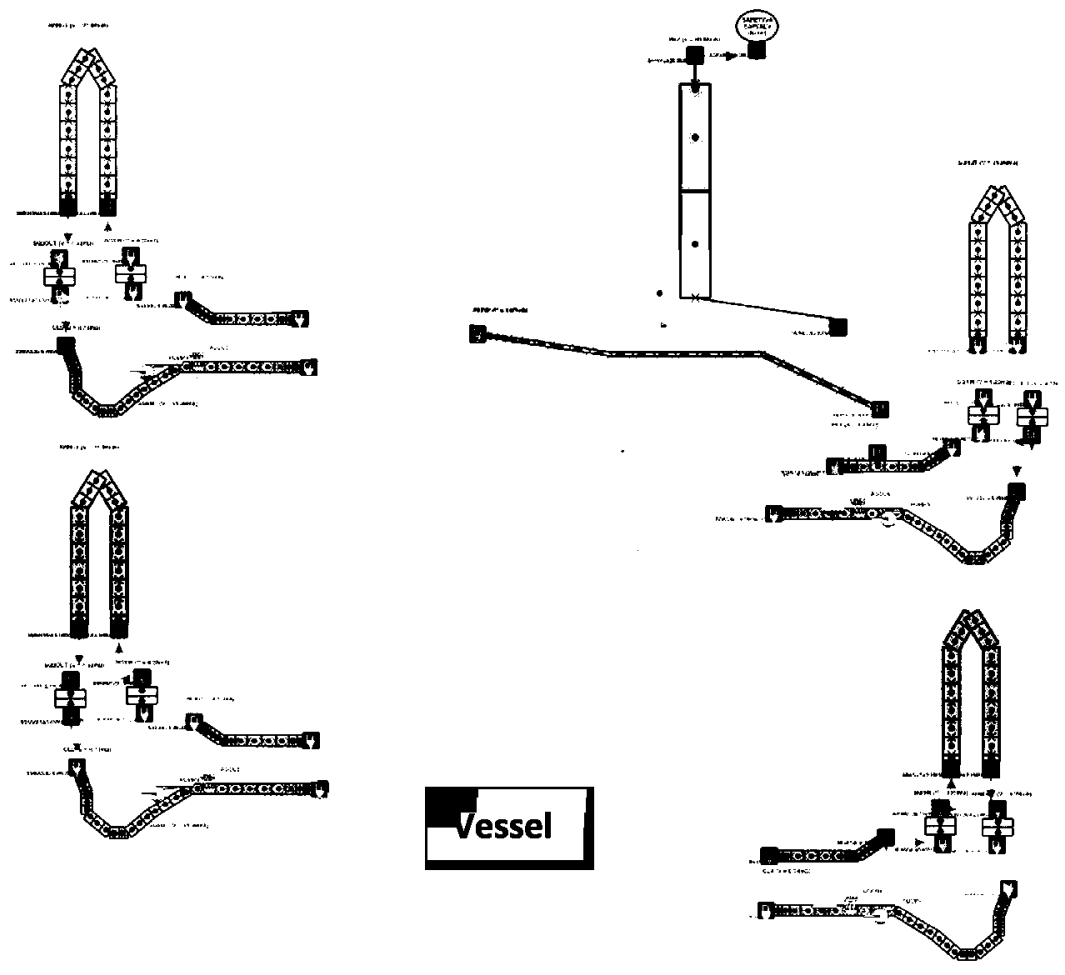

Fig. 4.6. CATHARE2 primary system model.

nodes for each assembly correspond to one thermal hydraulic channel in the FLICA-IV model.

\subsubsection{HZDR and UJV}

In the HZDR and UJV benchmark calculations the core is modeled using the DYN3D code. Due to its external coupling with ATHLET, DYN3D calculates both neutron kinetic and thermal hydraulic parameters of the core. The core model includes 193 flow channels (one channel per each of $141 \mathrm{UO} 2$ and 52 MOX fuel assemblies). In the neutronic part, the reactor core model is surrounded with an artificial band of assemblies representing radial and axial reflector. In the axial direction the core is divided into 34 axial layers ( 1 axial layer for lower reflector, 32 axial layers for heated core part and one axial layer for upper reflector). In the thermal hydraulic part, HZDR used one channel for the radial reflector while used UJV 64 channels. It means that in the latter case one coolant channel corresponds to one reflector assembly.

\subsubsection{GRS}

For the needs of the internal coupling solution, an 11 channels core model was developed and included in the ATHLET plant model. The mapping scheme is represented in Fig. 4.9. This mapping scheme is specific to this particular transient. A superchannel models the $3 / 4$ of the core which is connected to the unaffected loops. A second superchannel models the remaining quarter of the core connected to the affected loop. Two core bypass channels corresponding to the superchannels are modeled. The assembly with the stuck-out control rod and the surrounding 8 assemblies are modeled separately. This mapping scheme was chosen in order to provide another solution than the $1: 1$ scheme used in the two other DYN3D/ATHLET models. Qualitatively good results were expected with this model. However, because the Doppler temperature feedback is smeared in the two superchannels, more conservative results are expected for the first power peak.
The mixing between the cold / hot legs is modeled as follow: All core channels are connected to a single upper plenum; this means that a full mixing is assumed for the hot legs. In the cold legs, the mixing is modeled in the four downcomer channels and four lower plenum branches.

\subsubsection{UPM}

UPM used a COBAYA3 input deck with a radial nodalization of one node per assembly with one peripheral layer of radial reflector nodes. The axial nodalization consisted of 32 nodes of $11.43 \mathrm{~cm}$ for the active length and one additional node of $21.42 \mathrm{~cm}$ at the top and at the bottom as reflector.

For the benchmark calculations UPM used the coupling of COBAYA3 to the COBRA-TF code. The coupling has been developed using Python scripts that are in charge of:

- adjusting the meshes of both codes as necessary to fit them in scale, position and angle

- managing the calls to carry out the different steps in steady state and transient calculations

- establishing the coupling scheme and the time steps used by each code

COBAYA3/COBRA-TF coupling has been performed in an explicit way allowing each code using its optimum time step. While COBRA-TF calculates its own step using Courant limit and others, in COBAYA3 has to be imposed by the user based on experience. Several thermal hydraulic steps were performed within one neutron kinetic step. In order to fit the time for exchanging data, the last thermal hydraulic step is reduced to fit the time corresponding to the neutron kinetic interval.

The COBRA-TF input deck developed by GRS and provided to UPM includes one thermal hydraulic channel per assembly and 32 axial nodes per channel. Time dependent thermal-hydraulic 


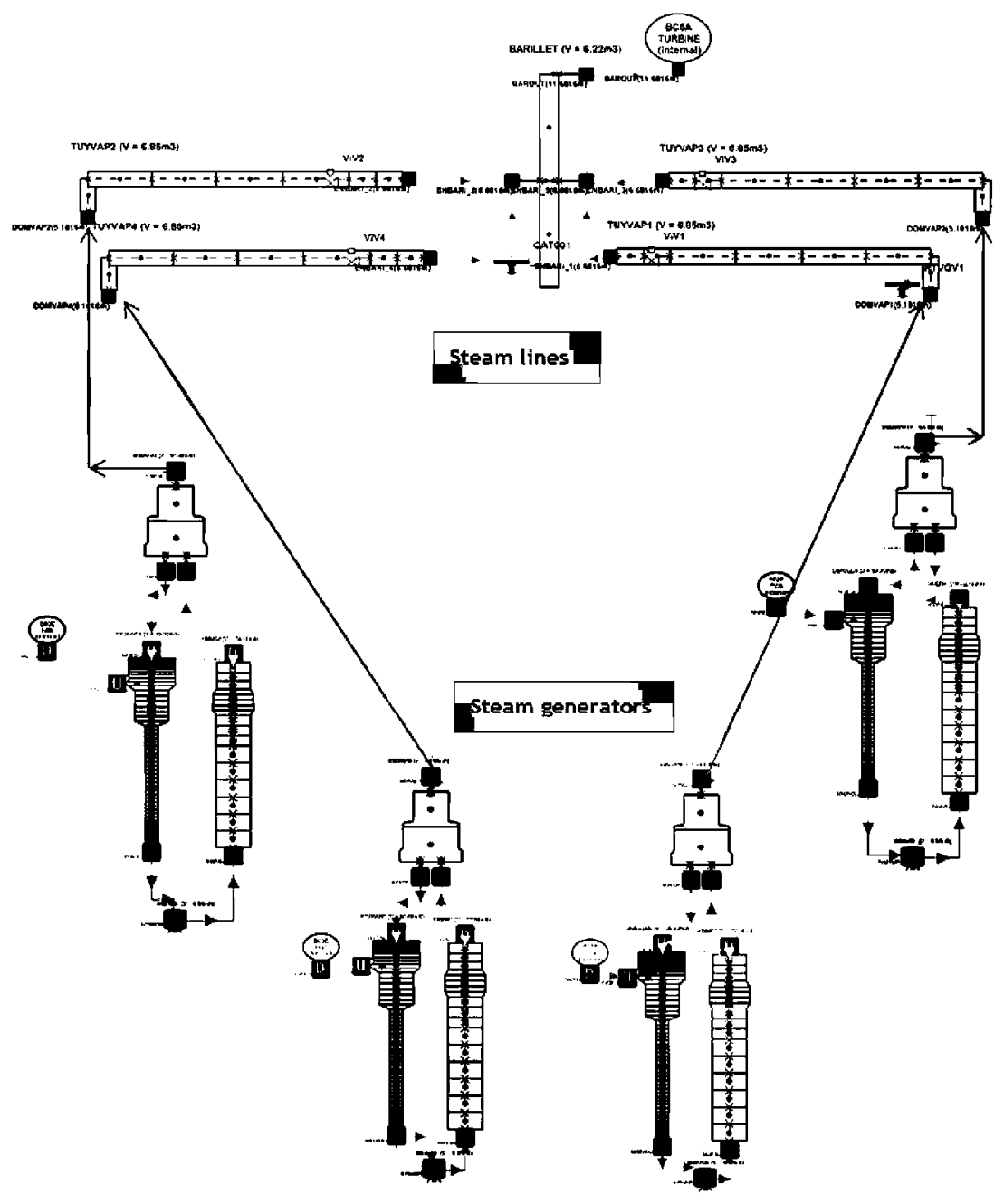

Fig. 4.7. CATHARE2 secondary system model.

boundary conditions at the inlet/outlet sections of the core obtained with DYN3D/ATHLET and supplied by HZDR has been used to simulate the plant behavior.

\subsubsection{PSI}

The PSI model using S3K is based on a nodalization including 193 radial channels and 32 axial layers, with channel inlet thermal hydraulic boundary conditions provided by HZDR. The transient TH boundary conditions required by S3K at the core inlet/outlet consisted in time-dependent channel inlet flow/temperatures and core exit pressure. A challenge for the implementation in the core model of these input data is that the base boundary conditions specification model of S3K, namely the Cold Leg Weighting (CLW) model, initially did not allow for temporal variations of the core-wide inlet temperature mixing distributions. Thus, an in-house version of S3K has been developed with a modified CLW subroutine such as to allow for individual channel inlet boundary conditions.

The standard methodology employed at PSI is based on the CASMO/SIMULATE code system. The basic principle is that the 2-

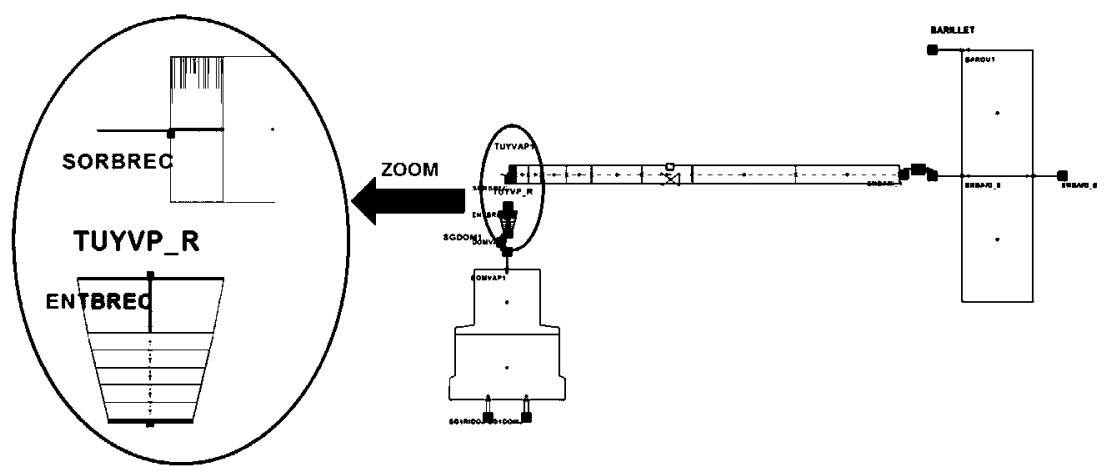

Fig. 4.8. CATHARE2 break modeling. 


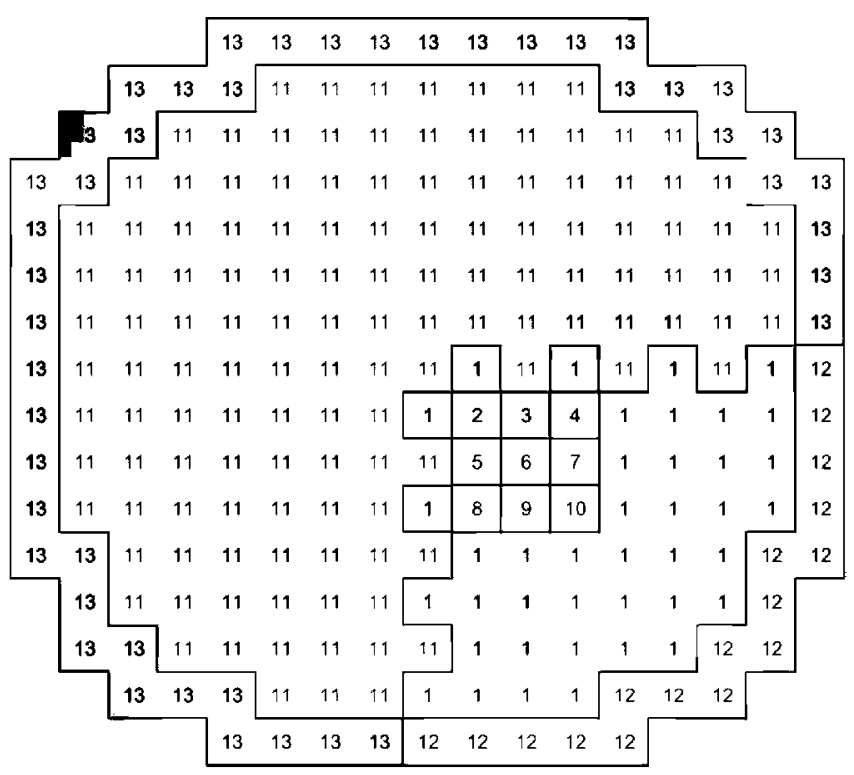

Fig. 4.9. Mapping scheme of neutron kinetic and thermal hydraulic channels in the GRS solution.

D assembly code CASMO, along with an appropriate multi-group neutron data library, is used to prepare the homogenized XS library for the subsequent 3D core analyses with SIMULATE and S3K for steady-state and transient analyses, respectively. However, in this benchmark, a procedure has been implemented in order to allow S3K to employ the XS library provided by the benchmark (see section 4.1). The implemented procedure consisted in converting the NEMTAB data into equivalent CASMO models and to combine this with an initialization of the SIMULATE macroscopic depletion model at the reference state conditions of the CASMO equivalent models. As for the kinetics data, the S3K model, which is based on 6 delayed neutron groups, could not use delayed neutron data specified in the benchmark ( 8 groups). Therefore, since no weighting scheme for group collapsing was provided in the benchmark specifications, the kinetics data in the NEMTAB X-S could not be used and instead, the nominal kinetics data from CASMO were applied.

In order to initialize the S3K model at the specified $-2 \$$ subcriticality level at ARI-1, a special procedure had to be formulated, since the code version employed for this benchmark (2.04.00_MOX4_PSI02) renormalizes the fission X-S to the initial steady-state k-eff before proceeding with the transient part of the simulation. Thus, an initial control rods pattern has been devised to allow reaching $-2 \$$ after full insertion of the $\mathrm{N}-1$ control rods clusters very shortly after steady-state initiation. Special attention has been taken in finding an initial control rods pattern that does not alter too significantly the initial flux distributions and resulting k-eff bias, compared to those from the ARI-1 configuration with this model. It is understood that this approach differs from that of other participants (e.g. no re-normalization of the initial k-eff from the steady-state solution, or addition of a constant source term to the time-dependent neutron flux equations), and thus could contribute to some of the differences discussed in thereafter section 5 .

\section{Comparison of results}

\subsection{Steady-state calculations}

In order to check the consistency of models and libraries used with the various NURESIM platform codes for the MSLB transient benchmark, several steady state calculations have previously been performed.

\subsubsection{Configurations compared}

Steady state configurations correspond to the initial state described in Section 2, at end of cycle and HZP conditions. Three different Control Rod positions have been tested to check that their calculated impact on reactivity and power distribution was coherent between participants. The corresponding configurations are designated ARO (All Rods Out), ARI (All Rods In), and ARI-C3 (All Rods Inserted except the heaviest one at position C3). The axial burnup profile of fuel assemblies, as specified for the initial state of the transient, is divided in 8 axial zones with burnup ratios ranging from 0.84 to 1.05 with respect to the mean value. This peaked burnup profile has been used in steady state calculations, however a flat one was also tested to check the correctness of the treatment of the interface with upper and lower reflectors.

The same nodal cross sections library, as described in 4.1 , was used, together with the three core neutronics simulators integrated in NURESIM platform, namely COBAYA, CRONOS2 and DYN3D, as described in 3.3, for these steady state comparisons.

\subsubsection{Discontinuity factors}

The XS library contains two sets of IDF (Interface Discontinuity Factors) to deal with the equivalence between heterogeneous transport and homogeneous diffusion. The first one is based on generalized equivalence theory, while the second is based on a black box homogenization with Selengut normalization. The choice of equivalence model may have particularly important impact in or around fuel assemblies with control rods inserted, which is why their influence on steady state results is then investigated by comparing calculations using either type of IDF, or without using any IDF. These three options are respectively designated GET, SEL and NOIDF.

The case of CRONOS2 core neutronics code is different from the situation of COBAYA3 and DYN3D. CRONOS2 solver is based on a finite elements method, considering continuous fluxes at interfaces. This is why it uses SPH (SuPerHomogenization) coefficients instead of IDF to deal with the heterogeneous-homogeneous equivalence, whereas COBAYA3 and DYN3D solvers are based on a nodal method, which can deal with discontinuity factors for equivalence. Unfortunately, the NURISP APOLLO XS calculation scheme does not produce SPH factors and an incompatibility between this scheme and the current version of CRONOS2 prevented us from directly computing SPH factors by CRONOS2 from transport neutron fluxes and currents. Thus, CRONOS2 calculations were only performed and compared to others without IDF.

\subsubsection{Reactivity}

The main keff results obtained by participants for the considered steady state configurations (only with the peaked burnup profile) are shown in Table 5.1. It appears clearly that the choice of using GET or SEL IDF has a very small impact on reactivity (less than $60 \mathrm{pcm}$ with all rods inserted) but the difference between the results with and without IDF is rather important when control rods are inserted (respectively 540 and $340 \mathrm{pcm}$ in the ARI and ARI-C3 configurations). It has to be noticed that DYN3D and COBAYA3 results with GET IDF are in good agreement (less than 100 pcm discrepancies), and that CRONOS results are in excellent agreement with COBAYA3 results without IDF.

The $\mathrm{C} 3$ rod worth is increased when no IDF (for COBAYA3) or no equivalence factors (for CRONOS2) are used to take into account the homogenization approximation during the cross sections library computations. 
Table 5.1

Steady state reactivity results.

\begin{tabular}{|c|c|c|c|c|c|c|}
\hline Participant & UPM & & & IRSN & HZDR & UJV \\
\hline Neutron Kinetics Code & COBAYA3 & & & CRONOS2 & DYN3D & DYN3D \\
\hline IDF & GET & SEL & NOIDF & NOIDF & GET & GET \\
\hline Control Rods ARO & 1.06495 & 1.06494 & 1.06494 & 1.06487 & 1.06438 & 1.06408 \\
\hline Control Rods ARI & 0.98368 & 0.98314 & 0.97844 & 0.97846 & 0.98291 & 0.98272 \\
\hline Control Rods ARI-C3 & 0.99641 & 0.99605 & 0.99309 & 0.99307 & 0.99573 & 0.99554 \\
\hline $\begin{array}{l}\text { C3 worth } \\
\text { (ARI-C3/ARI, pcm) }\end{array}$ & 1294 & 1313 & 1497 & 1493 & 1304 & 1304 \\
\hline
\end{tabular}

\subsubsection{Axial power distribution}

Results obtained by participants for the axial power distribution of the ARI-C3 configuration are presented in Fig. 5.1, for the peaked burnup profile (upper curve, corresponding to the specified initial state of the transient) and for the flat burnup profile (lower curve). The agreement between all participants is fairly good, for both burnup profiles, showing that the models agree well and that the interfaces with lower and upper reflectors are correctly taken into account.

\subsubsection{Radial power distribution}

Fig. 5.2 shows the radial power distribution (2D core map) obtained by UPM with COBAYA3 and using GET IDF for the ARI$\mathrm{C} 3$ configuration (initial state of the transient). It shows that the power peak arises in two fuel assemblies adjacent to the stuck control rod. The computed value of this power peak is 7.9.

The radial power distributions obtained for the same configuration by HZDR and UJV with DYN3D using GET IDF are very close to the one obtained by UPM with COBAYA3, as shown by the map

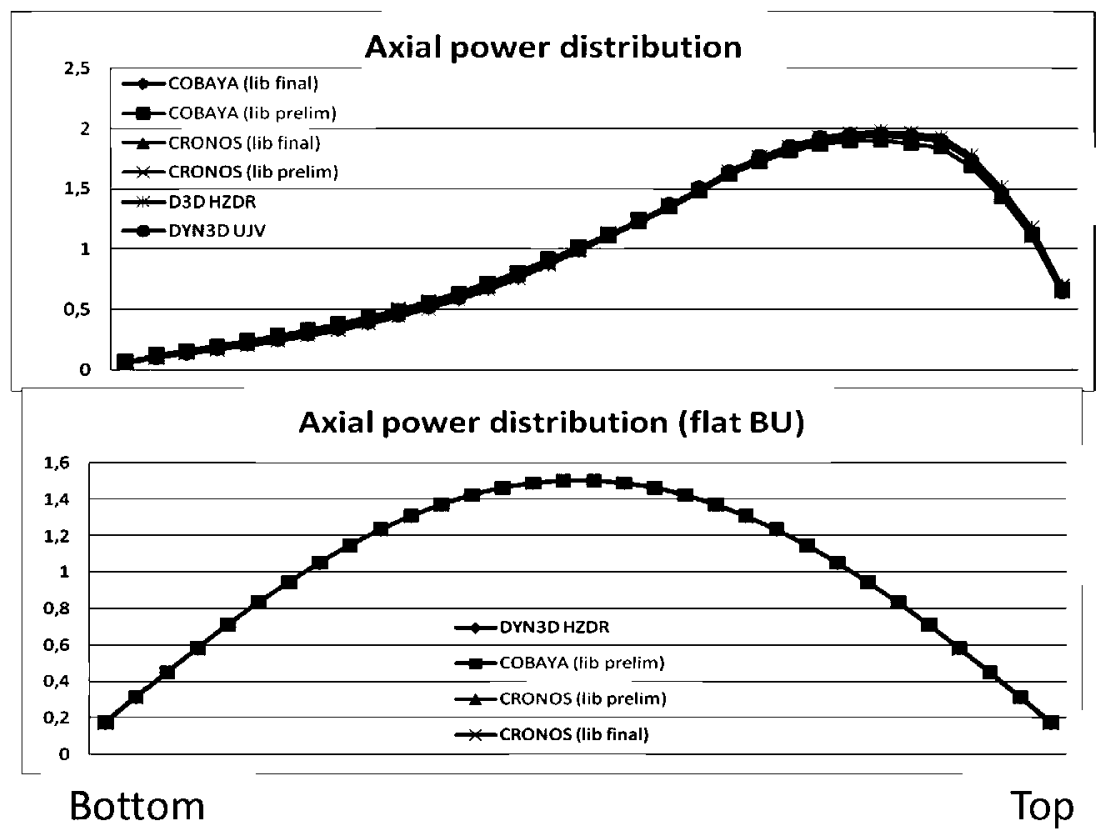

Fig. 5.1. Axial power distribution for either peaked or flat burnup profile.

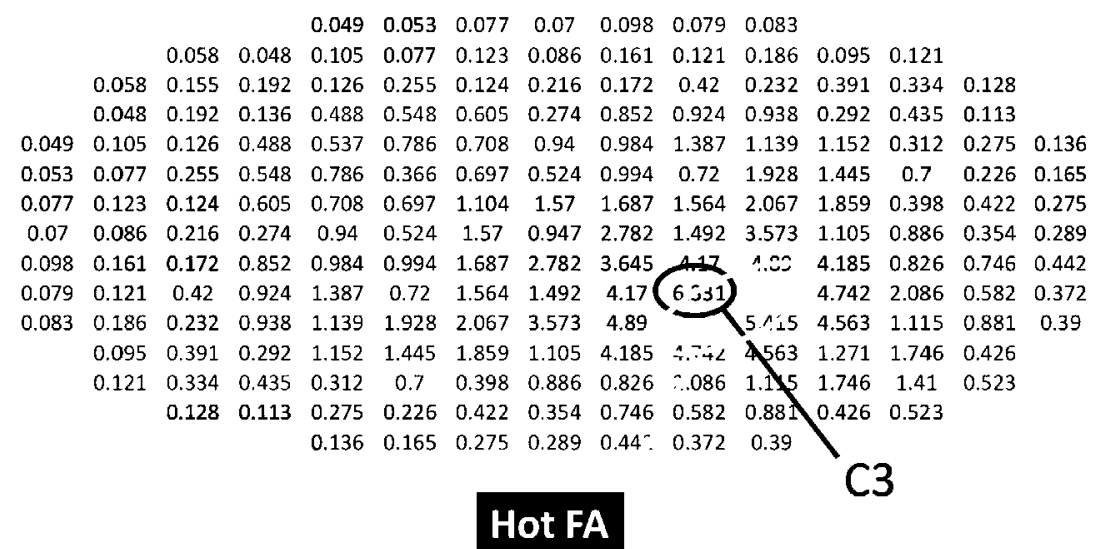

Fig. 5.2. Radial power distribution obtained by UPM (with COBAYA3 and GET IDF). 


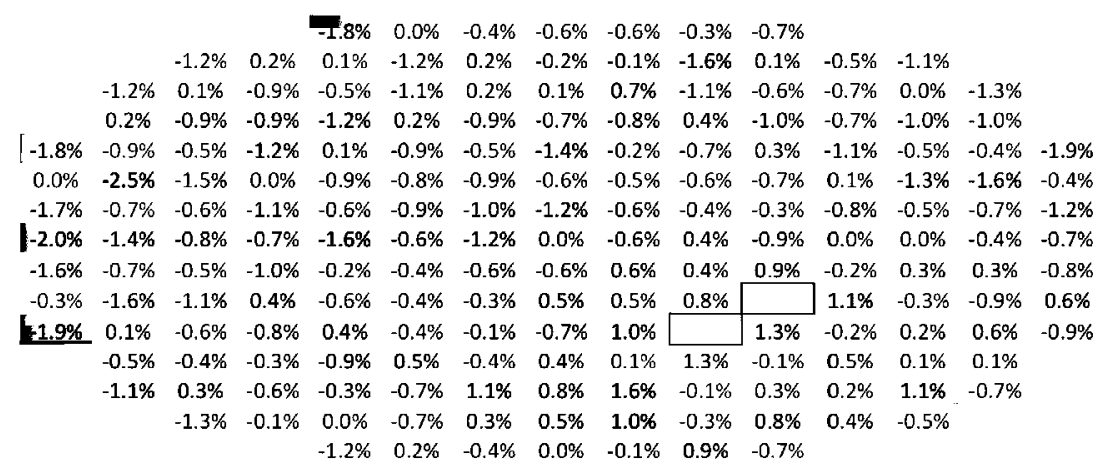

Fig. 5.3. Radial power discrepancies between HZDR (DYN3D) and UPM (COBAYA3) results.

presented in Fig. 5.3. The difference in the hot fuel assemblies is less than $0.5 \%$ and the maximum difference is $2.5 \%$.

Besides, calculations with COBAYA3 using SEL IDF instead of GET, exhibit a very small impact (less than $1 \%$ ) showing that both IDF models may be chosen, giving nearly the same results.

On the contrary, the radial power distribution obtained by IRSN with CRONOS2 reveals notable discrepancies ( $7 \%$ in hot fuel assemblies, until $10 \%$ in the hot sector and $22 \%$ in the periphery at the opposite of the stuck control rod). These discrepancies are, very probably, entirely due to the absence of SPH equivalence factors in the CRONOS2 cross sections library. Indeed, calculations with COBAYA3 without IDF give very close results to those by CRONOS2 (less than $0.5 \%$ in the hot sector and $1.5 \%$ in the whole core). The power peak obtained by IRSN is then overestimated by $7 \%$ with respect to UPM (COBAYA3), HZDR and UJV (DYN3D) results (Fig. 5.4).

\subsubsection{Conclusion of steady state calculations}

Comparison of steady state calculations by UPM (COBAYA3), HZDR and UJV (DYN3D), for reactivity, and axial and radial power distributions are in sufficiently good agreement to enable comparisons of transient calculations. As regards CRONOS2, the lack of equivalence factors to 'correct' the homogenized cross sections results in an increase not negligible discrepancy in the radial power distribution and (Table 5.1) in an increase of the rod worth, which will cause (compared to a 'true' case with SPH factors) a probable (but slight, the initial power discrepancy is not dramatic and we start from HZP) overestimation of the power transient.

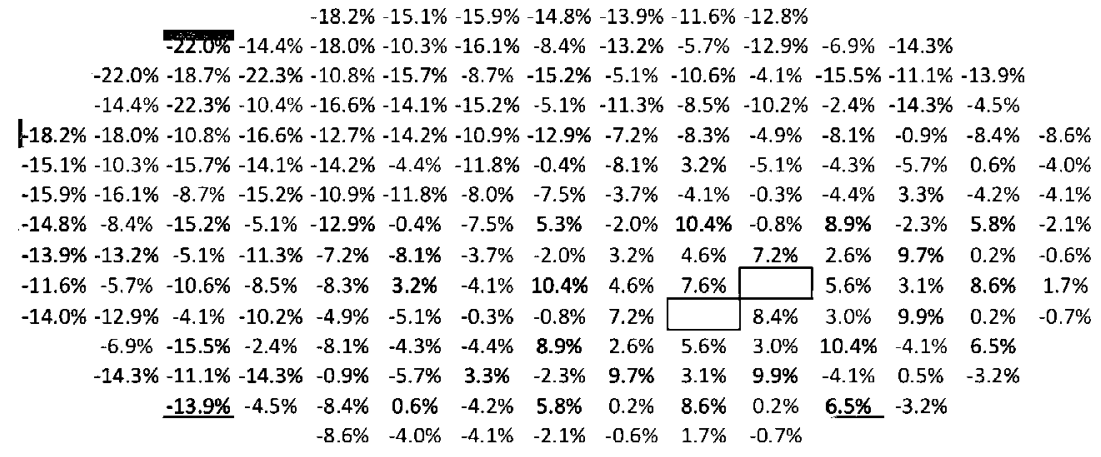

Fig. 5.4. Radial power discrepancies between IRSN (CRONOSNOIDF) and UPM (COBAYA GET IDF) results.

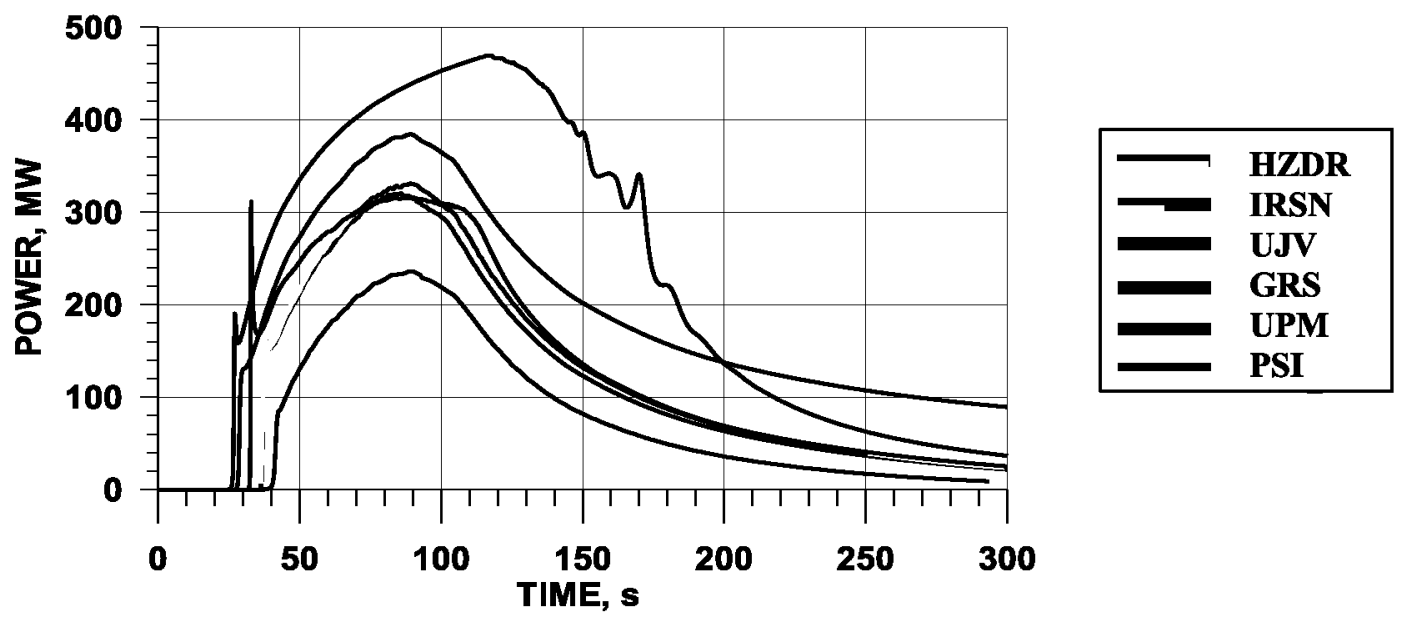

Fig. 5.5. Reactor power. 


\subsection{Transient calculations}

Figs. 5.5-5.8 show the comparison of reactor core parameters, most important of which are the reactor power (Fig. 5.5) and corresponding reactivity (Fig. 5.6). As can be seen from Fig. 5.5, all codes predict the re-criticality, the sudden rise of power after this re-criticality and the further smooth power increase which is later brought to sub-criticality, again. The spreading in time of reaching re-criticality is about $16 \mathrm{~s}$, and the level of power maximum itself differs by app. $230 \mathrm{MW}$. It should be noted that the presented power and reactivity behavior is supported by the other provided core parameters like average fuel temperature (Fig. 5.7) and average coolant density (Fig. 5.8). The reason for the observed differences in core parameters is discussed below. The differences in reactivity behavior between the HZDR and the UJV solution are so small that the curves cannot be distinguished in Fig. 5.6. It should be noted further that the single contributions of the reactivity as well as the average coolant density are not available from the UPM calculation.

The normalized axial power distributions at the moment of maximum reactor power are compared in Fig. 5.9. The maximum value varies from 1.5 (IRSN) to about 1.7 (HZDR, UJV, and PSI).

Fig. 5.10 shows the normalized radial power distribution in the core at the time point of maximum. Comparing the UJV and the HZDR results, the deviations are smaller than $3 \%$. The reason for this deviation is connected with the small time shift of the maxi- mum. The difference between HZDR and IRSN are much higher, they are going up to $15 \%$. Beside the shift in time here should be considered that the power level of both calculations differs by more than $100 \mathrm{MW}$. Furthermore here the influence of the secondary side plays an important role, which also reflects in the coolant temperature inlet distribution, where differences of up to $13.5 \mathrm{~K}$ are found. In the corresponding comparison between HZDR and UJV, the maximum differences are only $0.4 \mathrm{~K}$. In addition it has to be noted that the maximum deviations are located in the low power region.

All codes predict the same location of FAs with the highest power generation at the moment of maximum reactor power, but they give different estimations of the normalized power value for these assemblies (Fig. 5.10). The highest deviation in this parameter (14\%) is observed between the PSI and UPM results.

Figs. 5.11-5.13 show the behavior of the most important secondary side parameters. Three solutions are obtained using the ATHLET system code (HZDR, UJV and GRS) and one solution is obtained using the CATHARE2 (IRSN) system code. The secondary system parameters (as well as the primary system parameters below) are marked with the same colors as the core parameters in Figs. 5.4-5.10.

From the comparison it is to be seen that there are big differences between the ATHLET and CATHARE2 solutions. The driving force of this type of transient is the leak flow rate. Differences in the leak flow rate dominate all other parameters during the whole
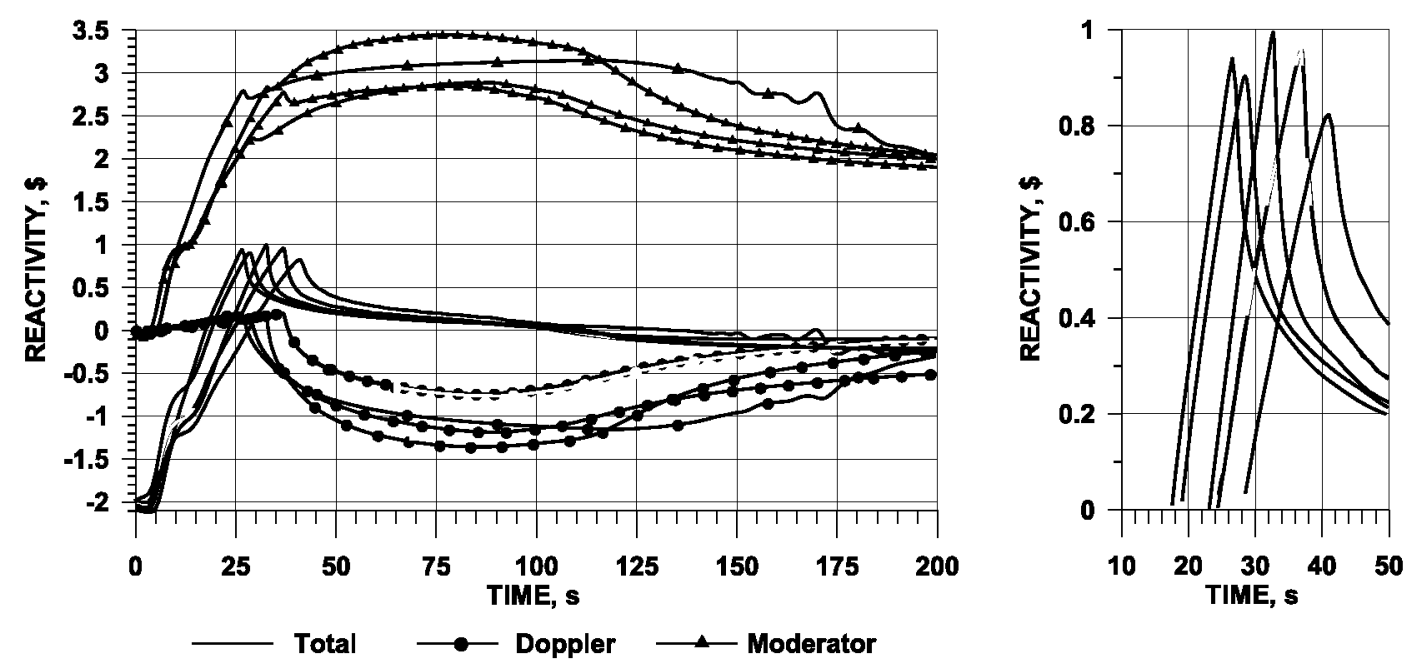

Fig. 5.6. Reactivity.

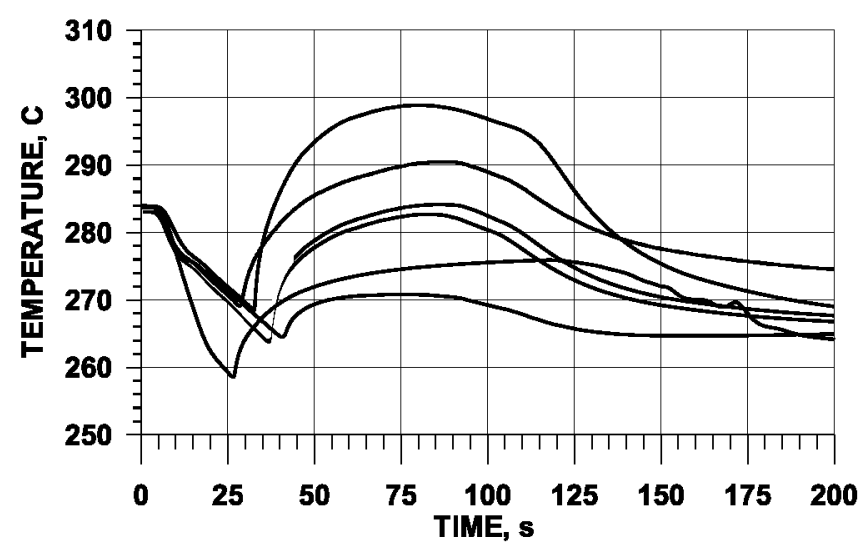

Fig. 5.7. Average fuel temperature.

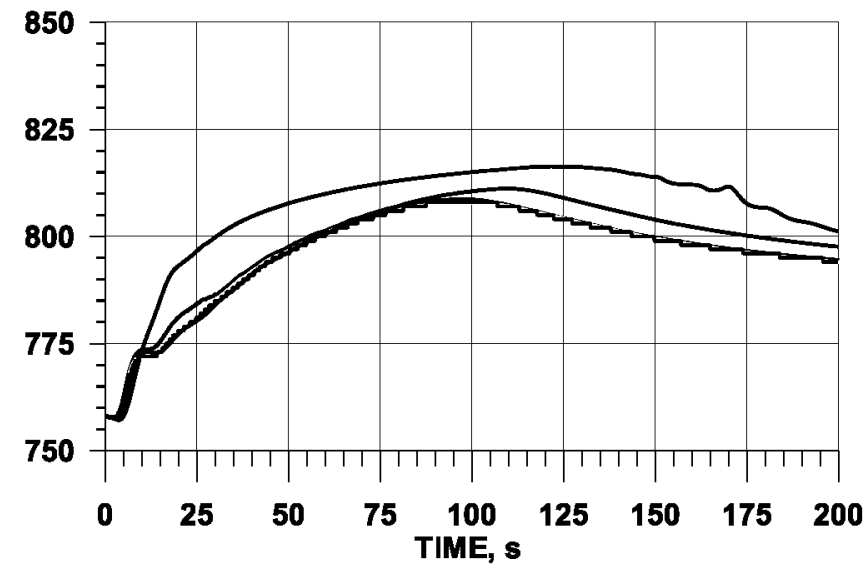

Fig. 5.8. Average coolant density. 


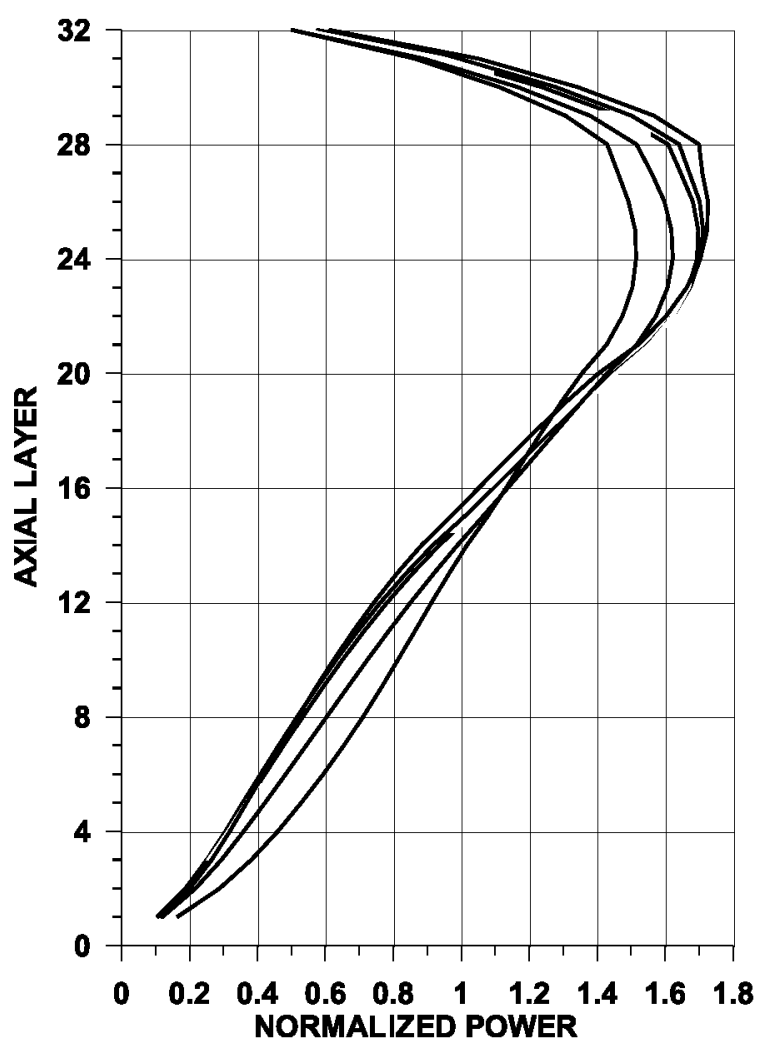

Fig. 5.9. Normalized axial power distributions.

transient. The IRSN calculation shows a higher total leak flow rate and, as a consequence, a faster depletion and depressurization of the secondary side (Figs. 5.11 and 5.12). It concerns the SGs both in the broken and intact loops (Figs. 5.12 and 5.13). These differ- ences in comparison to the ATHLET calculations are the reason for the differences in the above shown core parameters. It should be also mentioned that the steam separator model is not validated for such operational conditions.

Figs. 5.14-5.16 show the behavior of the most important primary side parameters. Again, small deviations between the results of the solutions using the ATHLET system code and considerable differences to the CATHARE2 solution are to be seen. The differences created at the secondary system are transferred to the primary system via the loop overcooling and cause finally the core behavior as reported above. One additional difference comes from a difference in modeling. In all ATHLET calculations, in the upper plenum there is a complete mixing of the coolant from all fuel assemblies. In the CATHARE2 calculation an incomplete mixing takes place in the upper plenum. This has indirect influence on the cold leg temperatures (Fig. 5.14) and, consequently, on the core inlet temperature distribution. Deeper cooling down of the primary system in the IRSN simulation results in significantly lower pressure and water level in the pressurizer compared with ATHLET predictions (HZDR, UJV, and GRS).

The timing of main events occurred during the accident is compared in Table 5.2. Only the core parameters are available for the comparison from UPM and PSI, because in these two calculations only the core model was used with the boundary conditions (core outlet pressure and assembly-wise distributions of the inlet mass flow rate and temperature) taken from the HZDR calculation performed with the coupled code DYN3D/ATHLET. The highest deviation in timing of the maximum core power $(30 \mathrm{~s})$ is observed between HZDR and IRSN predictions.

\section{Summary and conclusions}

The final objective of the PWR MSLB work package of the NURESAFE project is the so-called "high-fidelity" simulation of the transient using the couplings available on the NURESIM platform.

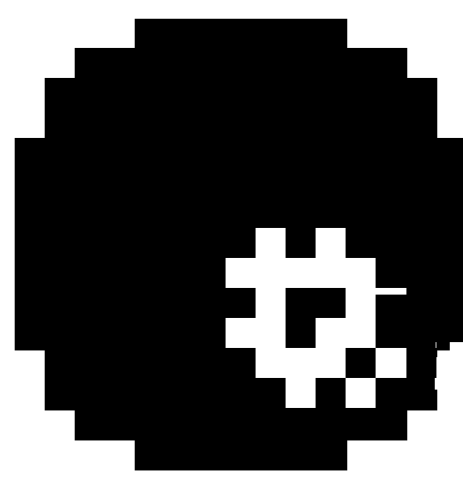

HZDR

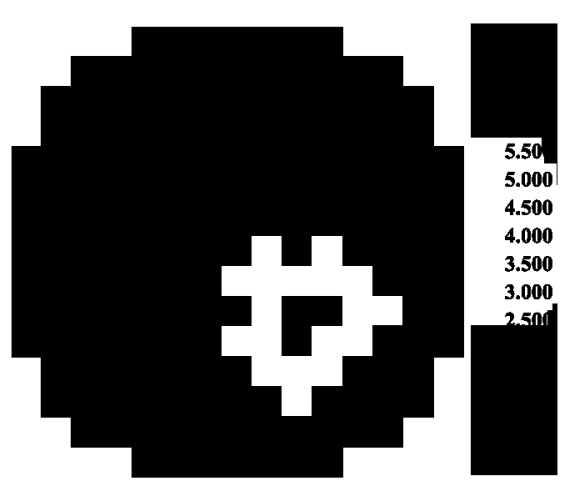

UPM
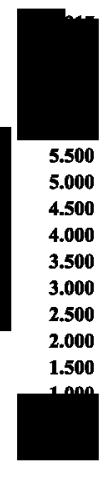

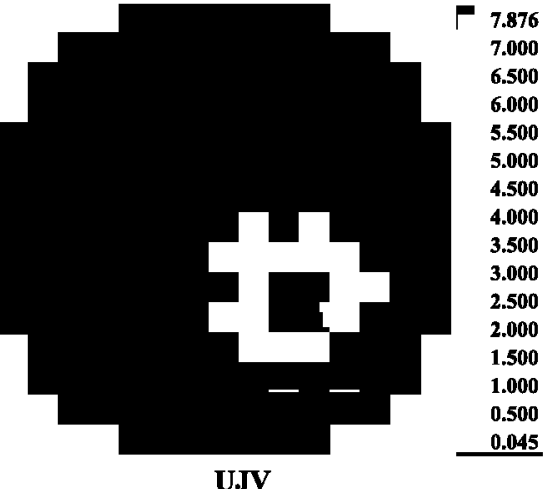

UJV

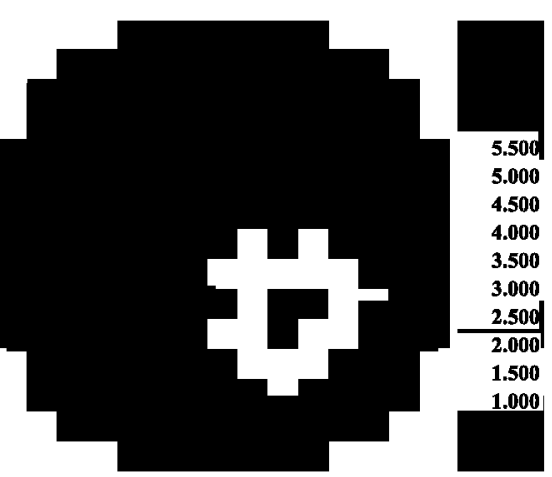

IRSN

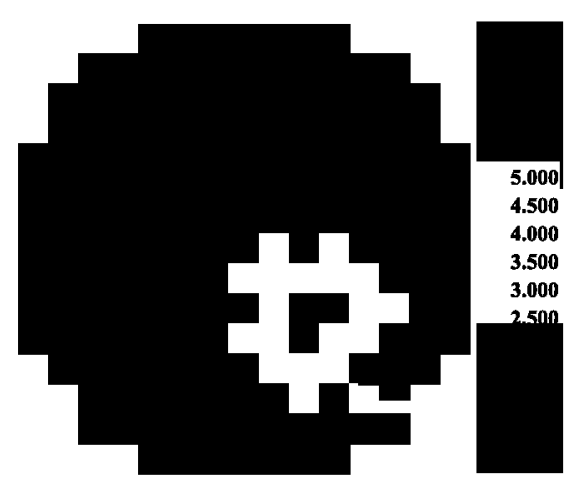

PSI

Fig. 5.10. Normalized radial power distributions at the moment of maximum reactor power. 

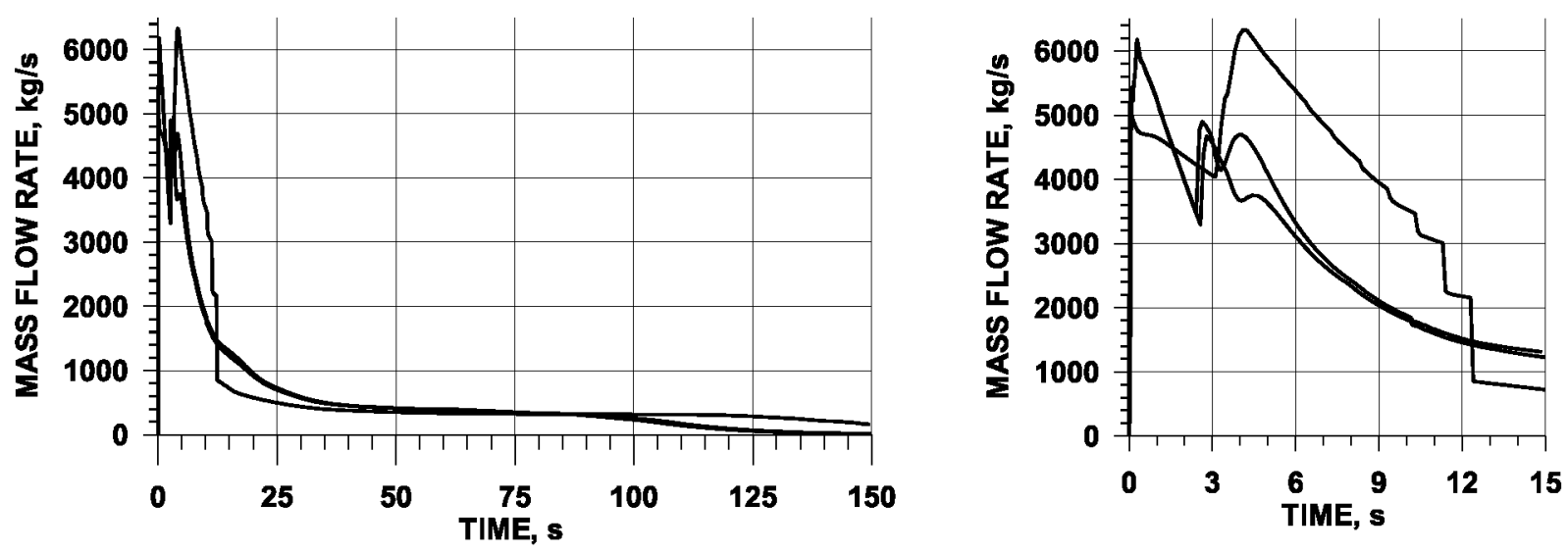

Fig. 5.11. Total (double-ended) discharge flow rate.
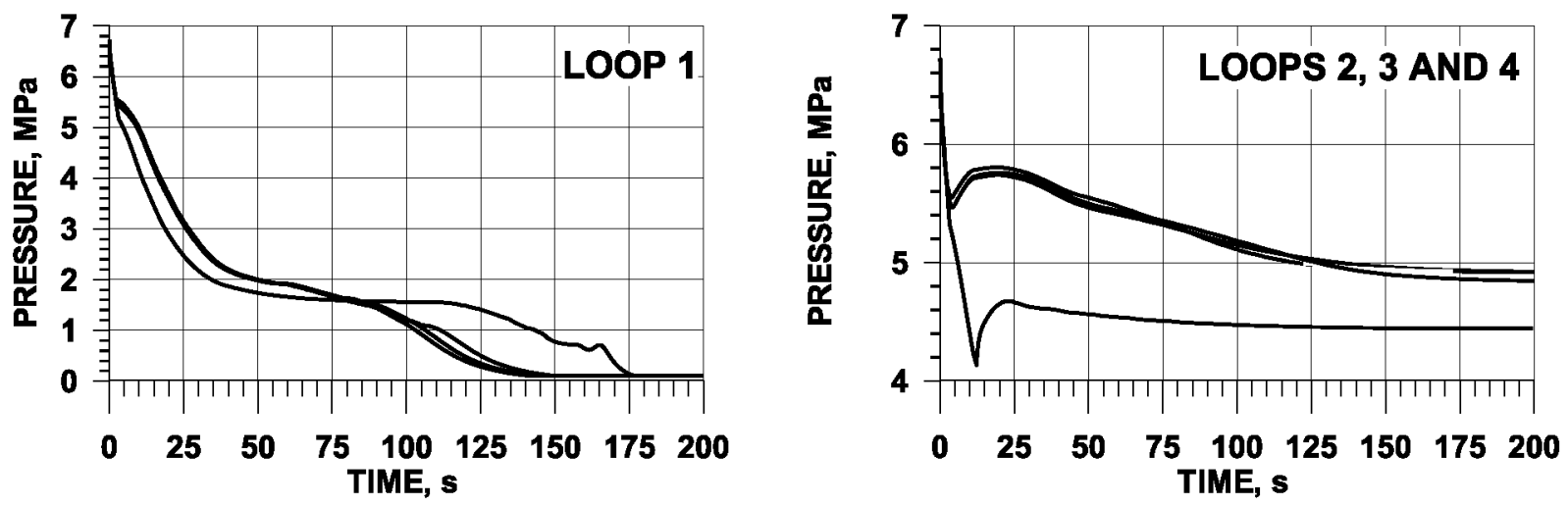

Fig. 5.12. SG pressure.
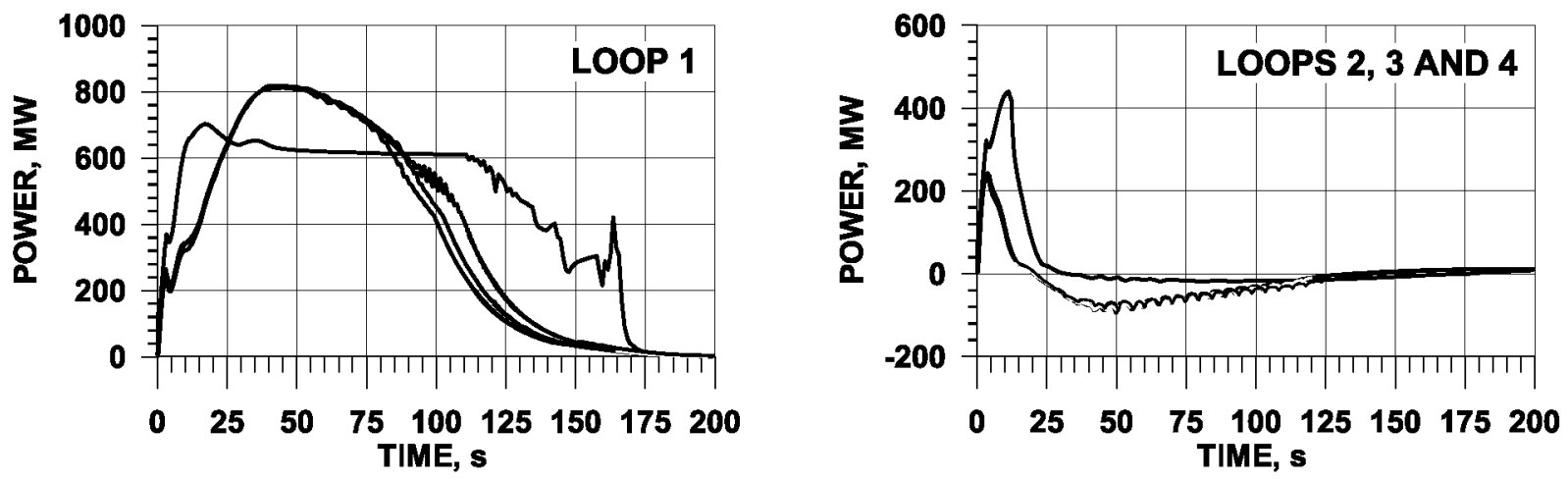

Fig. 5.13. SG power.

Before that, reference calculations using state-of-the-art simulation models are needed in order to show the influence of higherorder methods on the results.

The paper describes the results of the reference calculations for the MSLB benchmark defined in the frame of NURESAFE project. Six different solutions using different codes and code systems are provided for the comparison. The quantitative differences in the results are dominated by the differences in the secondary side parameters during the depressurization. The source of these differences comes mainly from the application of different models for the two-phase leak flow available in the different system codes. The use of two different thermal hydraulic system codes influences the results more than expected when the benchmark was created. 

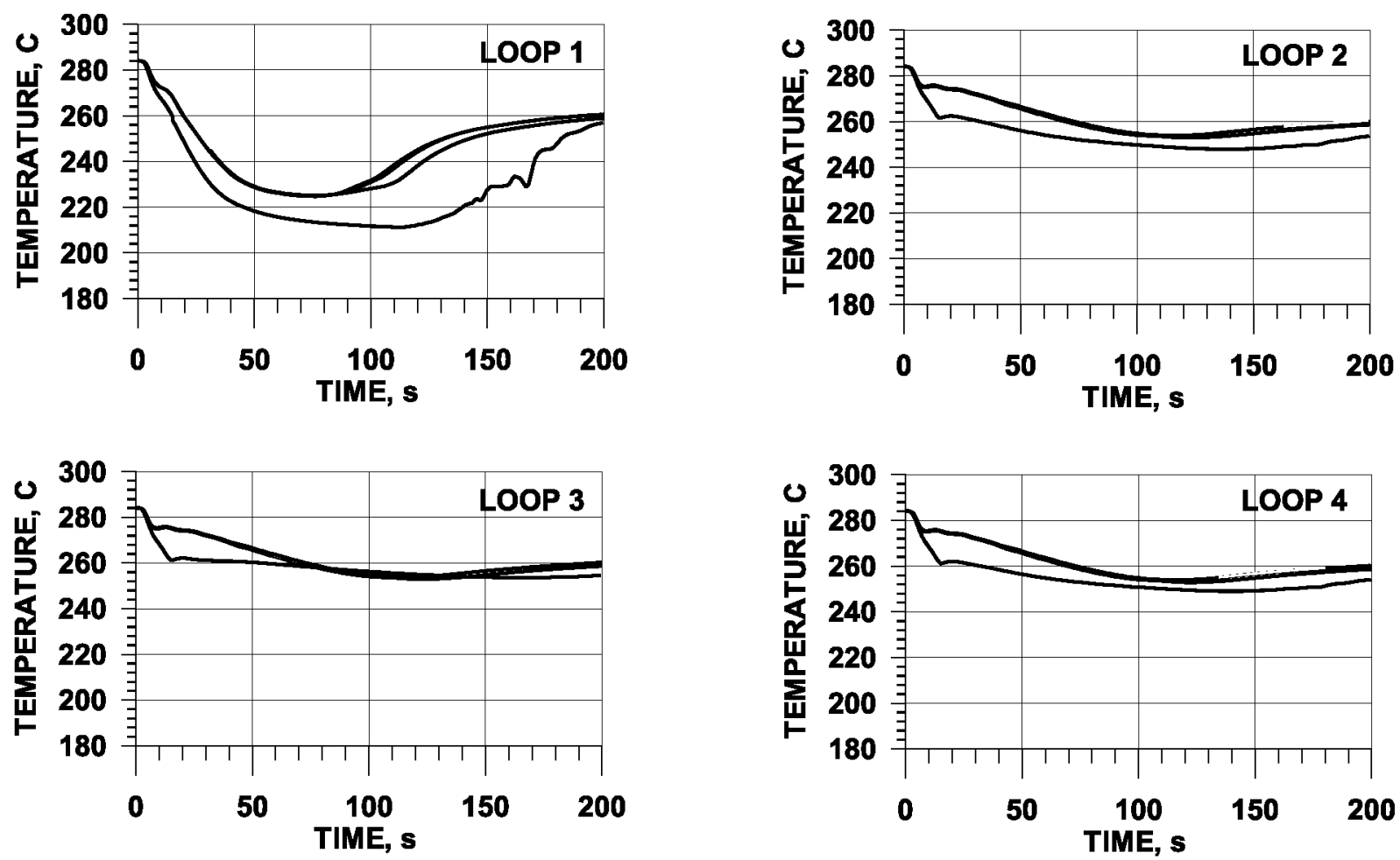

Fig. 5.14. Cold leg temperatures.

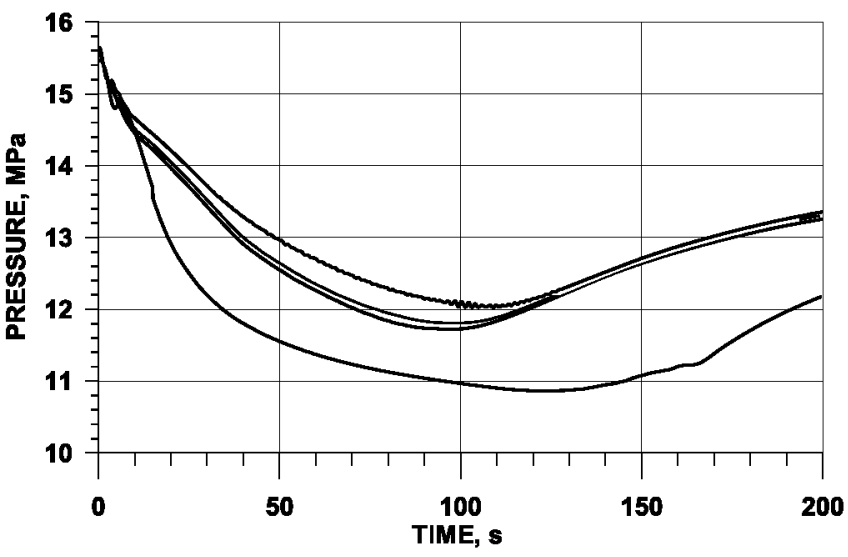

Fig. 5.15. Pressurizer pressure.

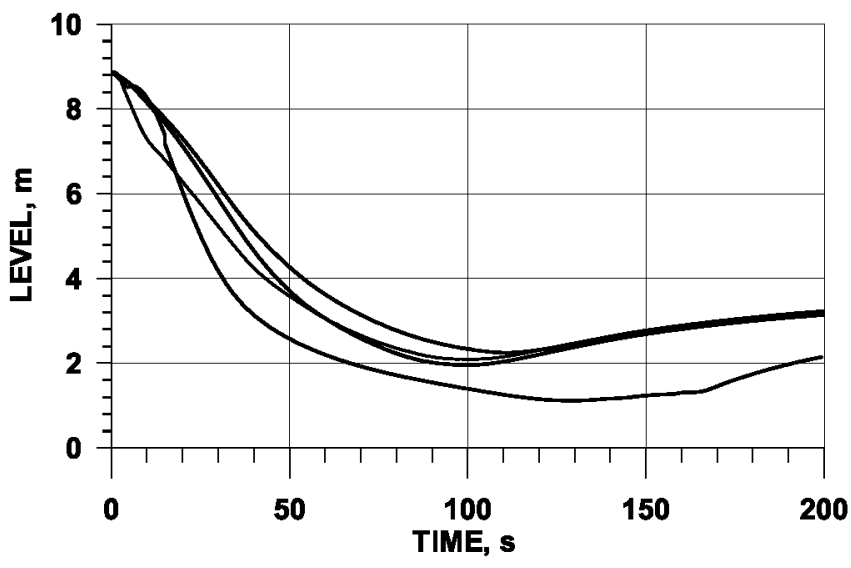

Fig. 5.16. Pressurizer water level.
Table 5.2

Sequence of events.

\begin{tabular}{ll}
\hline Event & $\begin{array}{l}\text { Time, s (IRSN/HZDR/UJV/GRS/ } \\
\text { UPM/PSI) }\end{array}$ \\
\hline Double-ended MSL break & $0.0 / 0.0 / 0.0 / 0.0 / 0.0 / 0.0$ \\
Start of FW supply to SGs & $0.0 / 0.0 / 0.0 / 0.0 /-/-$ \\
Core criticality reached & $17.5 / 24.3 / 24.0 / 23.0 / 27.8 / 18.9$ \\
Maximum overcriticality reached & $26.6 / 37.0 / 36.9 / 32.7 / 40.5 / 28.5$ \\
End of FW supply to SGs & $34.2 / 75.7 / 82.9 / 101.8 /-/-$ \\
Maximum core power reached & $116.8 / 85.8 / 89.4 / 88.1 / 89.0 / 88.8$ \\
Minimum primary pressure reached & $122.3 / 96.0 / 97.9 / 126.0 /-/-$ \\
Minimum coolant collapsed level in & $128.6 / 100.5 / 100.6 / 111.4 /-/-$ \\
$\quad$ pressurizer reached & \\
Return to subcritical state & $134.0 / 104.7 / 106.5 / 112.3 / 111.3 / 108.0$
\end{tabular}

The codes integrated into the NURESIM platform showed their applicability to a challenging transient like a main steam line break.

The present work is funded by the European Commission under the 7th EUROATOM Framework Program within the NURESAFE Project contract No. 323263.

Aragones, J.M., Ahnert, C., Garcia-Herranz, N., 2007. The analytic coarse-mesh finite difference method for multigroup and multidimensional diffusion calculations. Nucl. Sci. Eng. 157, 1-15.

Austregesilo, H., et al., 2012. ATHLET Mod. 3.0 Cycle A, Models and Methods, GRS P- 1, vol. 4, Rev. 3.

BEMUSE Phase IV Report (2008), Simulation of a LB-LOCA in ZION Nuclear Power Plant. Appendices A to D. NEA/CSNI/R(2008) 6/VOL2.

Bernard, F., Sánchez-Cervera, S., Garcia-Herranz, N., 2014. Nodal-level XS library parametrized for MSLB, NURESAFE, D12.23, May 2014.

Bestion, D., 2017. Multiscale Simulation of Light Water reactor thermalhydraulics. In: M\&C 2017 - International Conference on Mathematics \& Computationa 
Methods Applied to Nuclear Science \& Engineering, Jeju, Korea, April 16-20, 2017, on USB.

Chanaron, B. et al., 2015. Advanced multi-physics simulation for reactor safety in the framework of the NURESAFE project. Ann. Nucl. Energy 84, 166-177.

Chauliac, C. et al., 2011. NURESIM - a European simulation platform for nuclea reactor safety: Multi-scale and multi-physics calculations, sensitivity and uncertainty analysis. Nucl. Eng, Dsgn. 241, 3416-3426.

Feltus, M.A., 1994. Coupled 3-D kinetics thermal-hydraulic analysis of Hot Zero Power main steam line breaks using RETRAN and STAR codes. Nucl. Eng. Des. $146,439-450$.

Gaston, D. et al., 2009. MOOSE: a parallel computational framework for coupled systems of nonlinear equations. Nucl. Eng. Des. 239, 1768-1778.

Geffraye, G., Antoni, Q., Farvacque, M., Kadri, D., Lavialle, G., Rameau, B., Ruby, A., 2011. CATHARE2 V2.5.2: a single version for various application. Nucl. Eng. Des. $241,4456-4463$.

Grahn, A., Gommlich, A., Kliem, S., Bilodid, Y., Kozmenkov, Y., 2017. Coupling of the 3D neutron kinetic core model DYN3D with the CFD software TRIO_U and simulation of an MSLB scenario. Nucl. Eng. Des. 315, 117-127.

Grandi, G. (2011), SIMULATE-3K: Models and Methodology. Studsvik Scandpower Report, SSP-98/13.

Grundmann, U., Lucas, D., Rohde, U., Coupling of the Thermohydraulic Code ATHLET with the Neutron Kinetic Core Model DYN3D. In: Proc. Int. Conf. on Mathematics and Computations, Reactor Physics and Environmental Analysis, v. 1, 257-263, April 30-May 5, 1995, Portland, Oregon, USA.

Ivanov, K., Todorova, N., Sartori, E., 2003. Using the OECD/NRC pressurized water reactor main steam line break benchmark to study current numerical and computational issues of coupled calculations. Nucl. Technol. 142, 95-115.

Jimenez, G., Herrero, J.J., Gommlich, A., Kliem, S., Cuervo, D., Jimenez, J., 2015. Boron dilution transient simulation analyses in a PWR with neutronics/thermalhydraulics coupled codes in the NURISP project. Ann. Nucl. Energy 84, 86-97.

Kereszturi, A. et al., 2003. Development and validation of the three-dimensional dynamic code-KIKO3D. Ann. Nucl. Energy 30, 93

Kliem, S. et al., 2007. Oualification of coupled 3D neutron kinetic/thermal hydraulic code systems by the calculation of main steam line break benchmarks in a NPP with VVE-440 reactor. Nucl. Sci. Eng. 157, 280.

Kliem, S., Suehnel, T., Rohde, U., Hoehne, T., Prasser, H.-M., Weiss, F.-P., 2008. Experiments at the mixing test facility ROCOM for benchmarking of CFD-codes. Nucl. Eng. Des. 238, 566-576

Kolev, N., Todorova, G., Magnaud, C. Damian, F., Bellier, P., 2011. Development of calculation schemes and generation of XS libraries with APOLLO2 at the noda level for CRONOS2 and DYN3D diffusion and SP3 analyses of PWR and VVER, NURISP SP1 D1.3.1. February 2011.
Kozlowski, T., Downar, T.J., 2003. OECD/NEA and U.S. NRC PWR MOX/UO2 core transient benchmark, Final specifications, Revision 2, Perdue University, Indiana, U.S.A

Kozmenkov, Y., Kliem, S., 2013. Full system ATHLET model qualified for MSLB. NURESAFE. D12.21

Kozmenkov, Y., Kliem, S., 2014. PWR MSLB specification report. NURESAFE. D12.11.

Lautard J.J., Schneider D., Buadron A.M., 1999. Mixed Dual Method for Neutronic Reactor Core Calculations in the CRONOS System, M\&C99, Madrid, September 1999.

Lewis E.E., 1999. Primal, Mixed and Hybrid Finite Elements for Neutronics Computations, M\&C99, Madrid, September 1999.

Lizorkin, M., Gordienko, P., Kalugin, M., Kotsarev, A., Oleksyuk, D., 2015. Development of codes and KASKAD complex. Kerntechnik 80, 314.

Lozano, J.-A., Garcia-Herranz, N., Ahnert, C., Aragones, J.M., 2008. The analytic nodal diffusion solver ANDES in multigroups for 3D rectangular geometry: development and performance analysis. Ann. Nucl. Energy 35, 2365-2374.

Lozano, J.-A., Jimenez, J., Garcia-Herranz, N., Aragones, J.M., 2010. Extension of the analytic nodal diffusion solver ANDES to triangular-Z geometry and coupling with COBRA-IIIc for hexagonal core analysis. Ann. Nucl. Energy 37, 380-388.

Manera, A., Rohde, U., Prasser, H.-M., van der Hagen, T.H.J.J., 2005. Modelling of flashing-induced instabilities in the start-up phase of natural-circulation BWRs using the code FLOCAL. Nucl. Eng. Des. 235, 1517.

Marguet, S., 2011. La physique des réacteurs nucléaires, Collection EDF R\&D, Editions Lavoisier Tec \& Doc, ISBN 978-2-7430-1105-5.

Mylonakis, A. et al., 2014. Multi-physics and multi-scale methods used in nuclear reactor analysis. Ann. Nucl. Energy 72, 104-119.

Rohde, U. et al., 2016. The reactor dynamics code DYN3D - models, validation and applications. Prog. Nucl. Energy 89, 170-190.

Rohde, U., 2001. The modelling of fuel rod behaviour under RIA conditions in the code DYN3D. Ann. Nucl. Energy 28, 1343.

Salko, R.K., Avramova, M.N., (2015), CTF Theory Manual, Penn State.

Sanchez, R., 1988. APOLLO2: a modular code for multigroup transport calculation. Nucl. Sci. Eng. 100, 352

Sănchez-Cervera, S. et al., 2014a. Optimization of multidimensional cross-section tables for few-group core calculations. Ann. Nucl. Energy 69, 226-237.

Sănchez-Cervera, S., García-Herranz, N., Herrero, José J., Cuervo, D., 2014b. Effects of cross sections tables generation and optimization on rod ejection transient analyses. Ann. Nucl. Energy 73, 387-391.

Toumi, I., Bergeron, A., Gallo, D., Royer, E., Caruge, D., 2000. FLICA-IV: a threedimensional two-phase flux computer code with advanced numerical methods for nuclear applications. Nucl. Eng. Des. 200, 139-155. 